\title{
Confronting Mueller-Navelet jets in NLL BFKL with LHC experiments at $7 \mathrm{TeV}$
}

\section{B. Ducloué, ${ }^{a}$ L. Szymanowski ${ }^{b}$ and S. Wallon ${ }^{a, c}$}

${ }^{a}$ Laboratoire de Physique Théorique, Bât. 210, Université Paris-Sud, CNRS, 91405 Orsay, France

${ }^{b}$ Theoretical Physics Division, National Centre for Nuclear Research (NCBJ), Hoza 69, 00-681 Warsaw, Poland

${ }^{c}$ UPMC Univ. Paris 06, faculté de physique, 4 place Jussieu, 75252 Paris Cedex 05, France

E-mail: bertrand.ducloue@th.u-psud.fr, Lech.Szymanowski@fuw.edu.pl, wallon@th.u-psud.fr

ABSTRACT: More than 25 years ago, Mueller Navelet jets were proposed as a decisive test of BFKL dynamics at hadron colliders. We here study this process at NLL BFKL accuracy, taking into account NLL corrections to the Green's function and to the jet vertices. We present detailed predictions for various observables that can be measured at LHC in ongoing experiments like ATLAS or CMS at $\sqrt{s}=7 \mathrm{TeV}$ : the cross-section, the azimuthal correlations and the angular distribution of these jets. For this purpose, we apply realistic kinematical cuts and binning, and study the dependence of our results with respect to several parameters. We then compare our results with those that can be obtained in a fixed order NLO treatment, and propose specific observables which could actually be used as a probe of BFKL dynamics.

KEYwords: QCD Phenomenology, Hadronic Colliders 


\section{Contents}

1 Introduction 1

2 Basic formulas for LL and NLL calculation 4

2.1 Kinematics and general framework 4

2.2 LL order 5

2.3 NLL order 5

2.4 Strong coupling, renormalization scheme and PDFs at NLL 7

$\begin{array}{ll}2.5 & \text { Choice of scale } s_{0}\end{array}$

$\begin{array}{lll}2.6 & \text { Collinear improvement } & 7\end{array}$

3 Binning in $\left|\mathrm{k}_{J}\right| \quad \mathbf{8}$

3.1 Integration over $\left|\mathbf{k}_{J}\right| \quad 8$

$\begin{array}{lll}3.2 & \text { Energy-momentum conservation issues } & 9\end{array}$

4 Results: symmetric configuration $\quad 10$

$\begin{array}{lll}4.1 \text { Cross-section } & 11\end{array}$

$\begin{array}{lll}4.2 & \text { Azimuthal correlations } & 11\end{array}$

$\begin{array}{lll}4.3 & \text { Azimuthal distribution } & 16\end{array}$

5 Results: asymmetric configuration $\quad 19$

$\begin{array}{llr}5.1 \text { Cross-section } & 19\end{array}$

$\begin{array}{lll}5.2 & \text { Azimuthal correlations } & 19\end{array}$

$6 \quad$ Limit of small-R cone $\quad 22$

$\begin{array}{lll}7 & \text { Conclusions } & 23\end{array}$

\section{Introduction}

One of the important longstanding theoretical questions raised by QCD is its behaviour in the perturbative Regge limit $s \gg-t$. Based on theoretical grounds, one should identify and test suitable observables in order to test these peculiar dynamics.

First, one should select processes in which the presence of a hard scale justifies the use of perturbative QCD. At high energy, QCD is a massless theory with vector bosons, and it has two kinds of infrared (IR) divergences, namely the soft and the collinear divergences. For sufficiently inclusive quantities, both kinds of divergences cancel. Still, they are responsible for large logarithms, which may compensate the smallness of the strong coupling. At leading order, the soft singularities manifest themselves as powers of $\alpha_{S} \ln s /|t|$, resummed by the leading logarithmic (LL) BFKL Pomeron [1-4]. The collinear singularities are responsible for large logarithms of ratios of the transverse scales, which are resummed at leading logarithmic order (LLQ) by the DGLAP equation [5-8]. 


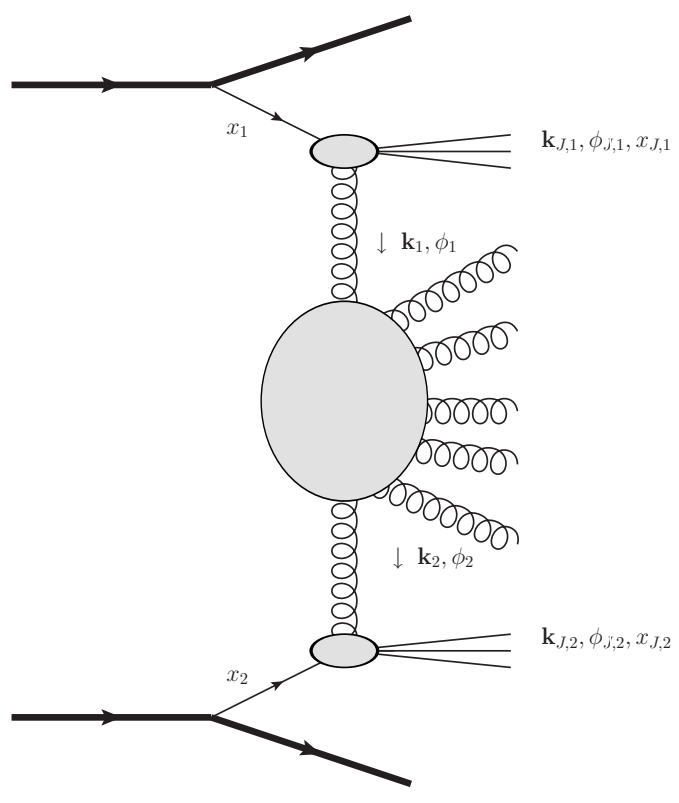

Figure 1. Kinematics.

The Regge limit is expected to be governed by the soft perturbative dynamics of QCD, which we want to reveal, and not by its collinear dynamics. The key point is thus to select processes in which the hard collinear scales are of similar magnitude, in such a way that the difference between a fixed order calculation and a collinear resummed result should be tiny, while a BFKL type of resummation should modify the predictions dramatically.

During the last 25 years, there have been many attempts to see manifestations of BFKL resummation effects. In inclusive DIS at HERA [9-11] or in total $\gamma^{*} \gamma^{*}$ cross-section at $e^{+} e^{-}$ colliders [12-18], the hard scale is the $\gamma^{*}$ virtuality. Exclusive processes have also been proposed and studied, either for heavy meson production $(J / \Psi, \Upsilon)$, the hard scale being provided by the meson mass [19-22], or meson electroproduction at large $t$ [21, 23], for which HERA data seems to favour a BFKL picture [24, 25]. At future high energy and high luminosity colliders like ILC, processes like $\gamma^{(*)} \gamma^{(*)} \rightarrow \rho \rho$ could be a realistic exclusive test of the hard Pomeron [26-31], with the planned detectors designed to cover the very forward region.

Jets have been proposed as a powerful tool in order to study BFKL dynamics, like diffractive high energy double jet production [32-34] as well as central jets $[35,36]$ in hadron-hadron collisions. In this paper, we focus on Mueller-Navelet jets [37]. This test of BFKL is based on the measure of two jets at large $p_{T}$ (hard scale), such that $s \gg p_{T}^{2} \gg$ $\Lambda_{\mathrm{QCD}}^{2}$, separated by a large rapidity $Y$, including possible activity between the two observed jets, as illustrated in figure 1 . The idea is to consider two jets of similar $p_{T}$ in order to minimize the effect of collinear resummation. From a lowest order treatment it is clear that these two jets should be back-to-back, in the very forward and very backward regions.

On the other hand, the expectation is that the large value of $Y=\ln \left(s / p_{T}^{2}\right)$ should examplify the effect of BFKL dynamics, due to possible emission of gluons between them (thus the Pomeron contributes there at $t=0$ at the level of the cross-section), leading 
to enhanced terms which sum up as $\sum\left(\alpha_{s} Y\right)^{n}$ (LL), $\alpha_{s} \sum\left(\alpha_{s} Y\right)^{n}$ (NLL) [38, 39], etc..., leading to a power-like rise for the cross-section.

Besides the cross-section a more exclusive observable within this process drew the attention, namely the azimuthal correlation between these jets $[40,41]$. The signal of a BFKL dynamics is a decorrelation of relative azimuthal angle between emitted jets when increasing $Y$. Indeed, while a fixed order calculation implies that the two jets would be emitted back-to-back, the fact that more and more (untagged) gluons can be emitted between them when increasing their relative rapidity should lead to a decorrelation of this relative azimuthal angle. Studies were made at LL [40-42], which overestimates this decorrelation by far. A better agreement with the data [43] could be obtained in the LL scenario using an event generator which takes into account in an exact way the energymomentum conservation, which is a subleading effect in a pure BFKL approach [44]. On the other hand, the (kinematically) modified LL BFKL approach [45], again based on LL jet vertices, could also provide some better agreement with the data.

At the same time, an exact fixed NLO $\left(\alpha_{s}^{3}\right)$ Monte Carlo calculation using the program JETRAD [46] lead to a too low estimate of the decorrelation, while the Monte Carlo NLO program HERWIG [47] was in perfect agreement with the data. It should be noted that this last treatment includes some Sudakov resummation effects, which might be important. The inclusion of such effects within a BFKL approach in an open problem which might be of interest for phenomenology. We leave this issue for further studies.

Starting from first principles from the point of view of Regge and Quasi-multi-Regge kinematics, NLL $[48,49]$ and collinear resummed NLL [50] studies (with LL jet vertices) have been performed, improving the situation with respect to pure LL BFKL, but still leading to a much stronger decorrelation than the one seen by the data.

In a previous work, we showed, based on a full NLL analysis [51], that contrarily to the expectation, the NLL corrections to the Green's function and to the jet vertices $[52,53]$ are of similar magnitude, based on a Mathematica code. We focused there on a center-ofmass energy $\sqrt{s}=14 \mathrm{TeV}$, and considered jets with fixed values of transverse momenta $\mathbf{k}_{J, i}$.

In the present paper, we pursue this study and make detailed predictions for observables to be extracted in the ongoing experiments ATLAS and CMS. Since experimental data are given in bins, it thus requires that we integrate $\mathbf{k}_{J, i}$ over a finite range. We also fix the center-of-mass energy to be $\sqrt{s}=7 \mathrm{TeV}$. In the study of the azimuthal correlations, we extend the use of the collinear resummation method to non-zero conformal spins. We then study in detail the azimuthal distribution of jets, which is directly experimentally accessible. Finally, we make a detailed comparison (with the same set of parameters) of our predictions with the NLO fixed order results based on the code used in ref. [54].

Our numerical predictions are based on a new FORTRAN code which allows us to perform more detailed studies of the dependency on various parameters (PDFs, renormalization/factorization scale, choice of $s_{0}$ scale). To check the consistency of our results, a detailed comparison has been made, in the mixed case of NLL BFKL Green's function and LL jet vertices $V^{(0)}$, with the previous studies of ref. [49, 50]. 


\section{Basic formulas for LL and NLL calculation}

\subsection{Kinematics and general framework}

We consider two hadrons (in practice protons) which collide at a center-of-mass energy $\sqrt{s}$ producing two very forward jets, whose transverse momenta are labeled by Euclidean two dimensional vectors $\mathbf{k}_{J, 1}$ and $\mathbf{k}_{J, 2}$, and by their azimuthal angles $\phi_{J, 1}$ and $\phi_{J, 2}$. The jet rapidities $y_{J, 1}$ and $y_{J, 2}$ are related to the longitudinal momentum fractions of the jets via $x_{J}=\frac{\left|\mathbf{k}_{J}\right|}{\sqrt{s}} e^{y_{J}}$. The two partons produced by each of these two hadrons, which initiate the hard process, are treated in a collinear way. For large $x_{J, 1}$ and $x_{J, 2}$, collinear factorization leads to a differential cross-section which reads

$$
\frac{\mathrm{d} \sigma}{\mathrm{d}\left|\mathbf{k}_{J, 1}\right| \mathrm{d}\left|\mathbf{k}_{J, 2}\right| \mathrm{d} y_{J, 1} \mathrm{~d} y_{J, 2}}=\sum_{\mathrm{a}, \mathrm{b}} \int_{0}^{1} \mathrm{~d} x_{1} \int_{0}^{1} \mathrm{~d} x_{2} f_{\mathrm{a}}\left(x_{1}\right) f_{\mathrm{b}}\left(x_{2}\right) \frac{\mathrm{d} \hat{\sigma}_{\mathrm{ab}}}{\mathrm{d}\left|\mathbf{k}_{J, 1}\right| \mathrm{d}\left|\mathbf{k}_{J, 2}\right| \mathrm{d} y_{J, 1} \mathrm{~d} y_{J, 2}},
$$

where $f_{\mathrm{a}, \mathrm{b}}$ are the parton distribution functions (PDFs) of a parton a (b) in the according proton, characterized by their longitudinal momentum fraction $x_{i}$. The hard process is then described using $k_{T}$-factorization. The logarithmically enhanced contributions are taken care of by convoluting, in transverse momentum space, the BFKL Green's function $G$ with the two jet vertices, according to

$$
\frac{\mathrm{d} \hat{\sigma}_{\mathrm{ab}}}{\mathrm{d}\left|\mathbf{k}_{J, 1}\right| \mathrm{d}\left|\mathbf{k}_{J, 2}\right| \mathrm{d} y_{J, 1} \mathrm{~d} y_{J, 2}}=\int \mathrm{d} \phi_{J, 1} \mathrm{~d} \phi_{J, 2} \int \mathrm{d}^{2} \mathbf{k}_{1} \mathrm{~d}^{2} \mathbf{k}_{2} V_{\mathrm{a}}\left(-\mathbf{k}_{1}, x_{1}\right) G\left(\mathbf{k}_{1}, \mathbf{k}_{2}, \hat{s}\right) V_{\mathrm{b}}\left(\mathbf{k}_{2}, x_{2}\right),
$$

where the Mandelstam variable $\hat{s}=x_{1} x_{2} s$ refers to the hard subprocess. The jet vertices $V_{a, b}$ were calculated at NLL order in ref. $[52,53]$. Combining the PDFs with the jet vertices, we can thus write

$$
\begin{aligned}
& \frac{\mathrm{d} \sigma}{\mathrm{d}\left|\mathbf{k}_{J, 1}\right| \mathrm{d}\left|\mathbf{k}_{J, 2}\right| \mathrm{d} y_{J, 1} \mathrm{~d} y_{J, 2}} \\
& \quad=\int \mathrm{d} \phi_{J, 1} \mathrm{~d} \phi_{J, 2} \int \mathrm{d}^{2} \mathbf{k}_{1} \mathrm{~d}^{2} \mathbf{k}_{2} \Phi\left(\mathbf{k}_{J, 1}, x_{J, 1},-\mathbf{k}_{1}\right) G\left(\mathbf{k}_{1}, \mathbf{k}_{2}, \hat{s}\right) \Phi\left(\mathbf{k}_{J, 2}, x_{J, 2}, \mathbf{k}_{2}\right),
\end{aligned}
$$

where

$$
\Phi\left(\mathbf{k}_{J, i}, x_{J, i}, \mathbf{k}_{i}\right)=\int d x_{i} f\left(x_{i}\right) V\left(\mathbf{k}_{i}, x_{i}\right) .
$$

In order to deal both with the cross-section and with the azimuthal decorrelation, it is convenient to define the coefficients

$$
\begin{aligned}
& \mathcal{C}_{m}\left(\left|\mathbf{k}_{J, 1}\right|,\left|\mathbf{k}_{J, 2}\right|, Y\right) \equiv \int d y_{1} d y_{2} \delta\left(y_{1}+y_{2}-Y\right) \int \mathrm{d} \phi_{J, 1} \mathrm{~d} \phi_{J, 2} \cos \left(m\left(\phi_{J, 1}-\phi_{J, 2}-\pi\right)\right) \\
& \quad \times \int \mathrm{d}^{2} \mathbf{k}_{1} \mathrm{~d}^{2} \mathbf{k}_{2} \Phi\left(\mathbf{k}_{J, 1}, x_{J, 1},-\mathbf{k}_{1}\right) G\left(\mathbf{k}_{1}, \mathbf{k}_{2}, \hat{s}\right) \Phi\left(\mathbf{k}_{J, 2}, x_{J, 2}, \mathbf{k}_{2}\right) .
\end{aligned}
$$

The differential cross-section then corresponds to $\mathcal{C}_{0}$ which reads

$$
\int d y_{1} d y_{2} \delta\left(y_{1}+y_{2}-Y\right) \frac{\mathrm{d} \sigma}{\mathrm{d}\left|\mathbf{k}_{J, 1}\right| \mathrm{d}\left|\mathbf{k}_{J, 2}\right| \mathrm{d} y_{J, 1} \mathrm{~d} y_{J, 2}}=\frac{\mathrm{d} \sigma}{\mathrm{d}\left|\mathbf{k}_{J, 1}\right| \mathrm{d}\left|\mathbf{k}_{J, 2}\right| d Y}=\mathcal{C}_{0},
$$

while the azimuthal decorrelation for fixed $\left(\left|\mathbf{k}_{J, 1}\right|,\left|\mathbf{k}_{J, 2}\right|, Y\right)$ is given by

$$
\langle\cos (m \varphi)\rangle \equiv\left\langle\cos \left(m\left(\phi_{J, 1}-\phi_{J, 2}-\pi\right)\right)\right\rangle=\frac{\mathcal{C}_{m}}{\mathcal{C}_{0}} .
$$




\subsection{LL order}

The jet vertex $V$ at lowest order just implements the fact that the jet is made of a single parton, of the same nature as the collinear parton initiating the hard process. It reads [52, 53]:

$$
\begin{aligned}
V_{\mathrm{a}}^{(0)}(\mathbf{k}, x)=h_{\mathrm{a}}^{(0)}(\mathbf{k}) \mathcal{S}_{J}^{(2)}(\mathbf{k} ; x), & h_{\mathrm{a}}^{(0)}(\mathbf{k})=\frac{\alpha_{s}}{\sqrt{2}} \frac{C_{A / F}}{\mathbf{k}^{2}} \\
\mathcal{S}_{J}^{(2)}(\mathbf{k} ; x) & =\delta\left(1-\frac{x_{J}}{x}\right)\left|\mathbf{k}_{J}\right| \delta^{(2)}\left(\mathbf{k}-\mathbf{k}_{J}\right) .
\end{aligned}
$$

In the definition of $h_{\mathrm{a}}^{(0)}, C_{A}=N_{c}=3$ is to be used for initial gluon and $C_{F}=\left(N_{c}^{2}-\right.$ 1) $/\left(2 N_{c}\right)=4 / 3$ for initial quark.

In the LL approximation, the BFKL kernel, because of its conformal invariance, is diagonalized by the eigenfunctions

$$
E_{n, \nu}\left(\mathbf{k}_{i}\right)=\frac{1}{\pi \sqrt{2}}\left(\mathbf{k}_{i}^{2}\right)^{i \nu-\frac{1}{2}} e^{i n \phi_{i}}
$$

with an eigenvalue given by

$$
\omega_{L L}(n, \nu)=\bar{\alpha}_{s} \chi_{0}\left(|n|, \frac{1}{2}+i \nu\right)
$$

with $\bar{\alpha}_{s}=N_{c} \alpha_{s} / \pi$ and

$$
\chi_{0}(n, \gamma)=2 \Psi(1)-\Psi\left(\gamma+\frac{n}{2}\right)-\Psi\left(1-\gamma+\frac{n}{2}\right),
$$

where $\Psi(x)=\Gamma^{\prime}(x) / \Gamma(x)$. Using this basis for both the Green's function and the jet vertices, one thus obtains, introducing the arbitrary (at LL) scale $s_{0}$,

$\mathcal{C}_{m}=\left(4-3 \delta_{m, 0}\right) \int d y_{1} d y_{2} \delta\left(y_{1}+y_{2}-Y\right) \int \mathrm{d} \nu C_{m, \nu}\left(\left|\mathbf{k}_{J, 1}\right|, x_{J, 1}\right) C_{m, \nu}^{*}\left(\left|\mathbf{k}_{J, 2}\right|, x_{J, 2}\right)\left(\frac{\hat{s}}{s_{0}}\right)^{\omega(m, \nu)}$,

where

$$
C_{m, \nu}\left(\left|\mathbf{k}_{J}\right|, x_{J}\right)=\int \mathrm{d} \phi_{J} \mathrm{~d}^{2} \mathbf{k} \mathrm{d} x f(x) V(\mathbf{k}, x) E_{m, \nu}(\mathbf{k}) \cos \left(m \phi_{J}\right)
$$

\section{$2.3 \quad$ NLL order}

At NLL, the jet can be made of either a single or two partons. The collinear singularities can be absorbed consistently in the renormalized PDFs, as was shown in refs. [52, 53], for a given infrared-safe jet algorithm. These jet vertices read symbolically

$$
V_{\mathrm{a}}(\mathbf{k}, x)=V_{\mathrm{a}}^{(0)}(\mathbf{k}, x)+\alpha_{s} V_{\mathrm{a}}^{(1)}(\mathbf{k}, x) .
$$

The explicit form for the NLL $V_{\mathrm{a}}^{(1)}$ are rather lengthy and are intimately dependent on the jet algorithm. They will not be reproduced here. ${ }^{1}$ In our study we will use the cone

\footnotetext{
${ }^{1}$ They can be found in ref. [51], as extracted from refs. [52, 53] after correcting a few misprints of ref. [52]. They have been recently reobtained in ref. [55].
} 
algorithm with a size of $R_{\text {cone }}=0.5$. Note that other jet algorithms can be used, which do not affect significantly our obtained results.

The main issue when dealing with NLL corrections is to treat the NLL BFKL kernel. The point here is to avoid dealing explicitly with two convolutions in transverse momentum space, between the jet vertices and the Green's function. In general this kind of convolution is very difficult to handle with for numerical evaluations. Instead we prefer to mimic the treatment used for LL studies and work in the $(n, \nu)$ space. One is thus looking for a convenient basis in order to deal with the NLL BFKL kernel. The functions (2.10) cannot be used in principle, since conformal invariance is now broken. Anyway, the action of the NLL BFKL kernel on these LL eigenfunctions has been calculated in ref. [56], and it turns out that $E_{n, \nu}$ are still eigenfunctions in an extended sense, if one now promotes the eigenvalue to become an operator containing a derivative with respect to $\nu[29,48$, 49]. When convoluting with jet vertices, this derivative acts on them, thus leading to a contribution to the eigenvalue which now depends on the jet vertices $[29,48,49,57]$

$$
\begin{aligned}
\omega_{N L L}(n, \nu)=\bar{\alpha}_{s} \chi_{0}\left(|n|, \frac{1}{2}+i \nu\right)+\bar{\alpha}_{s}^{2}\left[\chi_{1}\left(|n|, \frac{1}{2}+i \nu\right)\right. \\
\left.-\frac{\pi b_{0}}{2 N_{c}} \chi_{0}\left(|n|, \frac{1}{2}+i \nu\right)\left\{-2 \ln \mu_{R}^{2}-i \frac{\partial}{\partial \nu} \ln \frac{C_{n, \nu}\left(\left|\mathbf{k}_{J, 1}\right|, x_{J, 1}\right)}{C_{n, \nu}\left(\left|\mathbf{k}_{J, 2}\right|, x_{J, 2}\right)}\right\}\right],
\end{aligned}
$$

where

$$
\begin{aligned}
\chi_{1}(n, \gamma)= & \mathcal{S} \chi_{0}(n, \gamma)+\frac{3}{2} \zeta(3)-\frac{\beta_{0}}{8 N_{c}} \chi_{0}^{2}(n, \gamma) \\
& +\frac{1}{4}\left[\psi^{\prime \prime}\left(\gamma+\frac{n}{2}\right)+\psi^{\prime \prime}\left(1-\gamma+\frac{n}{2}\right)-2 \phi(n, \gamma)-2 \phi(n, 1-\gamma)\right] \\
& -\frac{\pi^{2} \cos (\pi \gamma)}{4 \sin ^{2}(\pi \gamma)(1-2 \gamma)}\left\{\left[3+\left(1+\frac{N_{f}}{N_{c}^{3}}\right) \frac{2+3 \gamma(1-\gamma)}{(3-2 \gamma)(1+2 \gamma)}\right] \delta_{n, 0}\right. \\
& \left.-\left(1+\frac{N_{f}}{N_{c}^{3}}\right) \frac{\gamma(1-\gamma)}{2(3-2 \gamma)(1+2 \gamma)} \delta_{n, 2}\right\}
\end{aligned}
$$

with the constant $\mathcal{S}=\left(4-\pi^{2}+5 \beta_{0} / N_{c}\right) / 12 . \quad \zeta(n)=\sum_{k=1}^{\infty} k^{-n}$ is the Riemann zeta function while the function $\phi$ reads

$$
\begin{aligned}
\phi(n, \gamma)=\sum_{k=0}^{\infty} & \frac{(-1)^{k+1}}{k+\gamma+\frac{n}{2}}\left(\psi^{\prime}(k+n+1)-\psi^{\prime}(k+1)\right. \\
& \left.\quad+(-1)^{k+1}\left[\beta^{\prime}(k+n+1)+\beta^{\prime}(k+1)\right]+\frac{\psi(k+1)-\psi(k+n+1)}{k+\gamma+\frac{n}{2}}\right),
\end{aligned}
$$

with

$$
\beta^{\prime}(\gamma)=\frac{1}{4}\left[\psi^{\prime}\left(\frac{1+\gamma}{2}\right)-\psi^{\prime}\left(\frac{\gamma}{2}\right)\right]
$$


At NLL accuracy, only the leading order vertex coefficients enter in the derivative term of (2.16), so that

$$
-2 \ln \mu_{R}^{2}-i \frac{\partial}{\partial \nu} \ln \frac{C_{n, \nu}^{(\mathrm{LO})}\left(\left|\mathbf{k}_{J, 1}\right|, x_{J, 1}\right)}{\left(C_{n, \nu}^{(\mathrm{LO})}\left(\left|\mathbf{k}_{J, 2}\right|, x_{J, 2}\right)\right)^{*}}=2 \ln \frac{\left|\mathbf{k}_{J, 1}\right| \cdot\left|\mathbf{k}_{J, 2}\right|}{\mu_{R}^{2}} .
$$

\subsection{Strong coupling, renormalization scheme and PDFs at NLL}

In this paper we will mainly use the MSTW 2008 PDFs [58]. We will make comparisons with several other sets of PDFs, as provided by the Les Houches Accord PDF Interface (LHAPDF) [59].

We use the two-loop strong coupling constant in the form

$$
\alpha_{s}\left(\mu_{R}^{2}\right)=\frac{1}{b_{0} L}\left(1+\frac{b_{1}}{b_{0}^{2}} \frac{\ln L}{L}\right),
$$

with $L=\ln \mu_{R}^{2} / \Lambda_{\mathrm{QCD}}^{2}$, and

$$
b_{0}=\frac{33-2 N_{f}}{12 \pi}, \quad \quad b_{1}=\frac{153-19 N_{f}}{24 \pi^{2}} .
$$

In the following, $\alpha_{s}$ or $\bar{\alpha}_{s}$ without argument is to be understood as $\alpha_{s}\left(\mu_{R}^{2}\right)$ or $\bar{\alpha}_{s}\left(\mu_{R}^{2}\right)$ respectively. The MSTW 2008 PDFs assume $\mu_{R}$ and $\mu_{F}$ to be equal. Therefore, we make the same identification everywhere in our analysis. The renormalization scale $\mu_{R}$ is chosen to be $\mu_{R}=\sqrt{\left|\mathbf{k}_{J, 1}\right| \cdot\left|\mathbf{k}_{J, 2}\right|}$.

\subsection{Choice of scale $s_{0}$}

At NLL, one should also pay attention to the choice of scale $s_{0}$. The choice $s_{0}=\sqrt{s_{0,1} s_{0,2}}$ with $s_{0, i}=\frac{x_{i}^{2}}{x_{J, i}^{2}} \mathbf{k}_{J, i}^{2}$ which we adopt is natural, since it does not depend on the momenta $\mathbf{k}_{1,2}$ to be integrated out. Besides, the dependence with respect to $s_{0}$ of the whole amplitude can be studied, when taking into account the fact that both the NLL BFKL Green's function and the vertex functions are $s_{0}$ dependent. We refer to section 3.2.2 of ref. [51] for a detailed discussion.

\subsection{Collinear improvement}

Several methods have been developed to improve the NLL BFKL Green's function for $n=0$, by imposing compatibility with the DGLAP equation [5-8] in the collinear limit [6063]. This is only required by the Green's function. Indeed, the collinear improvement deals with poles in the $\gamma$ plane ( $\gamma$ being a variable conjugated to transverse momentum in the Mellin transform). We have checked, based on a numerical study [51], that the jet vertices are free of $\gamma$ poles and thus do not call for any collinear improvement. In order to study the effect of such possible collinear improvement [60-63], a first attempt was performed in ref. [51], for $n=0$, using the scheme 3 of ref. [60]. Focusing on $n=0$ is enough for the study of the cross-section.

In view of the study of azimuthal correlation, a consistent treatment requires to take into account these collinear improvements also for $n \neq 0$. This has been investigated for the NLL BFKL Green's function in refs. $[49,50,57]$. We take into account these effects in this paper, thus improving the study of our previous work [51]. 


\section{Binning in $\left|\mathrm{k}_{J}\right|$}

\subsection{Integration over $\left|\mathrm{k}_{J}\right|$}

The experimental binning imposes that the values of $\left|\mathbf{k}_{J, i}\right|$ should be integrated in a given range. Each rapidity $y_{i}$ varies in the range $y_{\min } \leq y_{i} \leq y_{\max }$. In practice, we take $y_{\min }=0$ and $y_{\max }=4.7$. The total relative rapidity $Y=y_{1}+y_{2}$ which is experimentally accessible varies between 0 and 9.4. We will restrict ourselves to the region $Y \gtrsim 4$ (see discussion at the end of this section).

The phase space, at fixed $\left|\mathbf{k}_{J, 1}\right|,\left|\mathbf{k}_{J, 2}\right|, Y$, is defined as

$$
d(\mathrm{P} . \mathrm{S}) \equiv d\left|\mathbf{k}_{J, 1}\right| d\left|\mathbf{k}_{J, 2}\right| d y_{1} d y_{2} \delta\left(y_{1}+y_{2}-Y\right) .
$$

Correspondingly, the integration over the bin phase-space is defined as

$$
\int_{\text {bin }} d(\mathrm{P} . \mathrm{S}) \equiv \int_{k_{\min , 1}}^{k_{J} \max , 1} d\left|\mathbf{k}_{J, 1}\right| \int_{k_{J \min , 2}}^{k_{J} \max , 2} d\left|\mathbf{k}_{J, 2}\right| \int_{y_{\min }}^{y_{\max }} d y_{1} \int_{y_{\min }}^{y_{\max }} d y_{2} \delta\left(y_{1}+y_{2}-Y\right) .
$$

For a given observable $\mathcal{O}$, we thus define

$$
\mathcal{O}_{\text {bin }}=\int_{\text {bin }} d(\mathrm{P} . \mathrm{S}) \mathcal{O}
$$

which equivalently can be written as

$$
\begin{aligned}
& \mathcal{O}_{\text {bin }}=\int_{k_{J \min , 1}}^{k_{J \max , 1}} d\left|\mathbf{k}_{J, 1}\right| \int_{k_{J \min , 2}}^{k_{J \max , 2}} d\left|\mathbf{k}_{J, 2}\right| \int_{y_{\min }}^{y_{\max }} d y_{1} \int_{y_{\min }}^{y_{\max }} d y_{2} \delta\left(y_{1}+y_{2}-Y\right) \mathcal{O}\left(\left|\mathbf{k}_{J, 1}\right|,\left|\mathbf{k}_{J, 2}\right|, y_{1}, y_{2}\right) \\
& =\int_{k_{J \min , 1}}^{k_{J \max , 1}} d\left|\mathbf{k}_{J, 1}\right| \int_{k_{J \min , 2}}^{k_{J \max , 2}} d\left|\mathbf{k}_{J, 2}\right| \int_{y_{\min }}^{y_{\max }} d y_{1} \Theta\left(y_{\min } \leq Y-y_{1} \leq y_{\max }\right) \mathcal{O}\left(\left|\mathbf{k}_{J, 1}\right|,\left|\mathbf{k}_{J, 2}\right|, y_{1}, Y-y_{1}\right) .
\end{aligned}
$$

The resulting cross-section

$$
\left(\frac{d \sigma}{d Y}\right)_{\text {bin }}=\int_{\text {bin }} d(\text { P.S }) \frac{\mathrm{d} \sigma}{\mathrm{d}\left|\mathbf{k}_{J, 1}\right| \mathrm{d}\left|\mathbf{k}_{J, 2}\right| \mathrm{d} y_{J, 1} \mathrm{~d} y_{J, 2}}
$$

is in practice numerically evaluated by sampling each $y_{i}$ with a $\left(y_{\max }-y_{\min }\right) / 10=0.47$ binning. It thus means that in eq. (3.5), the $y_{1}$ integration is replaced by a discrete sum, which is then multiplied by a 0.47 width. This cross-section is shown in figure 2 , in the pure LL approximation as well as in the full NLL treatment. This figure shows that a very significant fraction $(\sim 80 \%)$ of the cross-section is obtained for $k_{J \max } \sim 60 \mathrm{GeV}$. We will further discuss this in the next subsection in relation with energy-momentum conservation issues.

As long as the jet vertices are treated in the LL approximation, the integration with respect to $\left|\mathbf{k}_{J, i}\right|$ can be performed analytically. As a consistency check, we compare, in the Tevatron kinematics used in ref. [49], the integration with respect to $\left|\mathbf{k}_{J, i}\right|$ with boundaries $k_{J \min , 1}=20 \mathrm{GeV}, k_{J \min , 2}=50 \mathrm{GeV}$ and $k_{J \max , 1}=k_{J \max , 2}=\infty$. Numerically, due to 


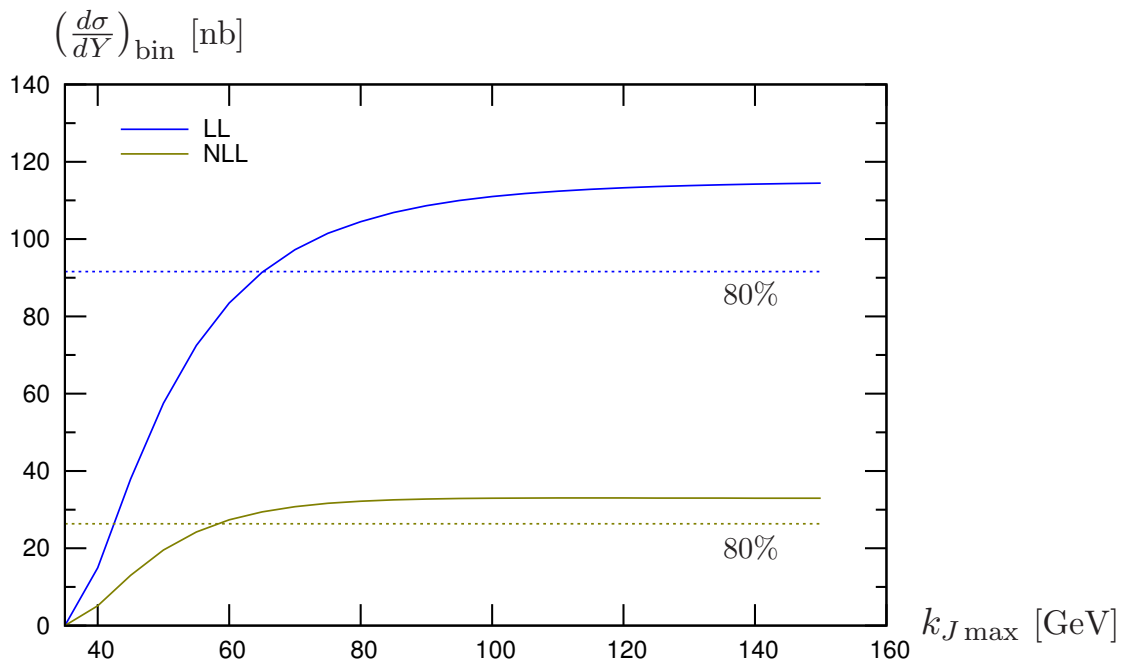

Figure 2. Growth of the cross-section with $k_{J \max }=k_{J \max , 1}=k_{J \max , 2}$, for $Y=6.6$ and $k_{J \min }=$ $k_{J \min , 1}=k_{J \min , 2}=35 \mathrm{GeV}$.

numerical instabilities when evaluating the Green's function for fixed values of $\left|\mathbf{k}_{J, i}\right|$ at low $Y$, the comparison with data is expected to be rather poor for $Y<\frac{\pi}{2 \alpha_{s} N_{c}}$, i.e. typically for $Y \lesssim 4$. A detailed study of this issue will be made elsewhere [64]. In the rest of this paper, we will restrict ourselves to the region $Y \gtrsim 4$.

To perform this comparison, we use the observable $\overline{\langle\cos \varphi\rangle}_{\text {bin }}$, which is the average of $\langle\cos \varphi\rangle$ on the experimental bin, that is defined here as (see eq. $(2.7))$ )

$$
\begin{aligned}
& k_{J \max , 1} k_{J \max , 2} \\
& \int^{\mathrm{d}} \mathrm{d}\left|\mathbf{k}_{J, 1}\right| \mathrm{d}\left|\mathbf{k}_{J, 2}\right| \mathcal{C}_{m} \\
& {\overline{\langle\cos (m \varphi)\rangle_{\mathrm{bin}}}}=\frac{k_{J \min , 1} k_{J \min , 2}}{k_{J \max , 1} k_{J \max , 2}} \\
& \int_{k_{J \min , 1}} \int_{k_{J \min , 2}} \mathrm{~d}\left|\mathbf{k}_{J, 1}\right| \mathrm{d}\left|\mathbf{k}_{J, 2}\right| \mathcal{C}_{0} \\
& =\frac{\int_{\text {bin }} d(\mathrm{P} . \mathrm{S})\langle\cos (m \varphi)\rangle \frac{\mathrm{d} \sigma}{\mathrm{d}\left|\mathbf{k}_{J, 1}\right| \mathrm{d}\left|\mathbf{k}_{J, 2}\right| \mathrm{d} y_{J, 1} \mathrm{~d} y_{J, 2}}}{\int_{\text {bin }} d(\mathrm{P} . \mathrm{S}) \frac{\mathrm{d} \sigma}{\mathrm{d}\left|\mathbf{k}_{J, 1}\right| \mathrm{d}\left|\mathbf{k}_{J, 2}\right| \mathrm{d} y_{J, 1} \mathrm{~d} y_{J, 2}}} .
\end{aligned}
$$

This comparison is shown in figure 3 , showing the consistency of our numerical results. ${ }^{2}$

\subsection{Energy-momentum conservation issues}

It is well known that the BFKL equation does not preserve energy-momentum conservation. However, this violation is expected to be smaller at higher order in perturbation theory,

\footnotetext{
${ }^{2}$ This comparison focuses on the Green's function. It thus assumes that the PDFs are equal to 1, with $x_{J, i}=1$. The scales $\mu_{F}$ and $\sqrt{s_{0}}$ are taken to be $\sqrt{k_{J \min , 1} k_{J \min , 2}}$, with $k_{J \min , 1}=20 \mathrm{GeV}$ and $k_{J \min , 2}=50 \mathrm{GeV}$.
} 


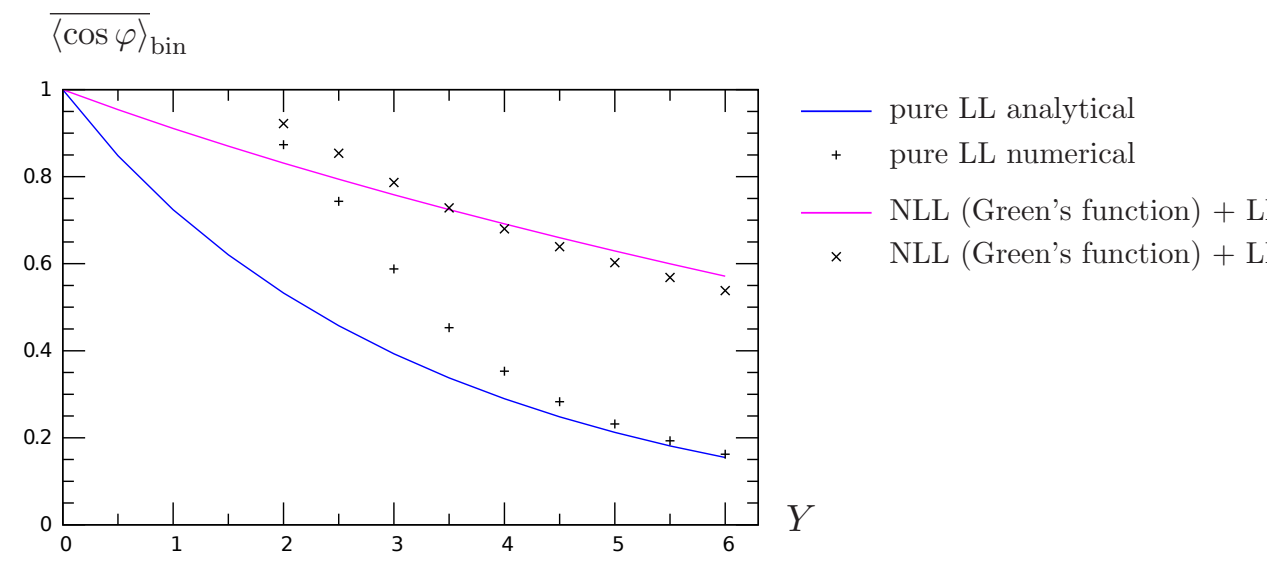

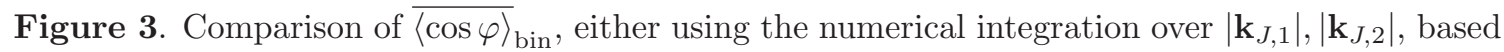
on our ForTRAn code (crosses), or the analytical integration (solid) as shown in ref. [49], for the Tevatron kinematics, for both pure LL BFKL and mixed NLL BFKL treatments.

i.e. when comparing NLL BFKL versus LL BFKL. In practice, one should thus avoid to use all the available collider energy. This means that one should satisfy the constraint

$$
y_{J, i} \ll \cosh ^{-1} \frac{x_{i} E}{k_{J, i}} .
$$

This implies that taking a lower $k_{J}$ provides a larger validity domain. This justifies in our opinion a strong experimental effort to extract low $k_{J}$ data.

In practice, with only a lower cut on $k_{J}$, one has to integrate over regions where the BFKL approach may not be valid anymore. For example, $k_{J}=60 \mathrm{GeV}$ leads to a constraint $y_{J, i} \ll 7.3$. For this reason it would be nice to have a measurement with bins including an upper cut on transverse momentum, $k_{J \min } \leq k_{J} \leq k_{J \max }$. Since the cross-sections are expected to be large, we believe that the statistics should be large enough to allow for a narrow binning in $k_{J}$, which should thus be mainly a detector issue. ${ }^{3}$ To conclude this section, we note that the $k_{J}$ integration reduces the $Y$ domain between jets. This $Y$ domain is also reduced by the $x_{i}$ integration, which is weighted by PDFs, strongly peaked at small $x_{i}$.

\section{Results: symmetric configuration}

In this section, we consider a symmetric configuration as planned to be studied by the CMS collaboration. We thus consider bins with cuts

$$
\begin{aligned}
35 \mathrm{GeV} & <\left|\mathbf{k}_{J, 1}\right|,\left|\mathbf{k}_{J, 2}\right|<60 \mathrm{GeV}, \\
0 & <y_{1}, y_{2}<4.7 .
\end{aligned}
$$

We consider several kinds of scenarios, starting from a pure LL approximation up to full NLL and collinear improved NLL approximations. The convention for colors is the same

\footnotetext{
${ }^{3}$ At CMS, a measurement with $k_{J \text { min }}=35 \mathrm{GeV}$ seems to be possible, while going down to $20 \mathrm{GeV}$ would probably require a dedicated trigger.
} 
in the whole paper:

$\begin{array}{ll}\text { blue: } & \text { pure LL result } \\ \text { magenta: } & \text { combination of LL vertices with pure NLL Green's function } \\ \text { green: } & \text { combination of LL vertices with collinear improved NLL Green's function } \\ \text { brown: } & \text { pure NLL result } \\ \text { red: } & \text { full NLL vertices with collinear improved NLL Green's function. }\end{array}$

\subsection{Cross-section}

We first consider the cross-section. The obtained results are displayed in figure 4 . Note that the Monte Carlo integration leads to a precision of the order of $2 \%$ to $5 \%$, which is too small to be seen in this figure.

This result confirms the fact that NLL corrections to the jet vertices are huge, of the same order of magnitude as the NLL corrections to the Green's function. The full NLL result leads to a cross-section which is significantly smaller than the one based on LL vertices combined with the pure NLL Green's function.

The curves obtained when combining the LL vertices with the pure NLL Green's function and when combining the LL vertices with the collinear improved NLL Green's function are almost indistinguishable. Similarly, the curves obtained when combining the NLL vertices with the pure NLL Green's function and when combining the NLL vertices with the collinear improved NLL Green's function are very close.

In figure 5, in the pure NLL case, we display the uncertainties due to the changes of the various involved parameters. The first effect which we study is the variation of the scales $s_{0}$ and $\mu_{F}$, as shown in figure $5(\mathrm{~L})$. The large uncertainty at very low $Y$ is related to the specific instabilities of NLL Green's function mentioned at the end of section 3.1, while the large $Y$ uncertainty is related to kinematical boundary effect (the cross-section almost vanishes).

The second effect, due to the dependency on the set of PDFs, is shown in figure $5(\mathrm{R})$. Note that we only display the pure NLL case, although the trend is similar for other scenarios.

Both of these effects are much smaller than the changes due to the NLL corrections to the jet vertices.

\subsection{Azimuthal correlations}

We now consider the azimuthal correlations. The obtained results are displayed in figure 6 , again using the color conventions (4.2). Note that the Monte Carlo integration leads to a precision of the order of a few $\%$ when using the NLL vertices, the numerical uncertainty being negligible in the case of LL jet vertices. We do not show it on this figure.

Comparing on one hand the pure LL scenario with the mixed LL vertex combined with the NLL Green's function, and the mixed LL vertex combined with the NLL Green's function with the full NLL treatment on the other hand, we see that the effect due to the modification of the jet vertex produces the largest correction. On the same plot, one can see the effect of collinear improvement. When including this effect for the whole set of the 


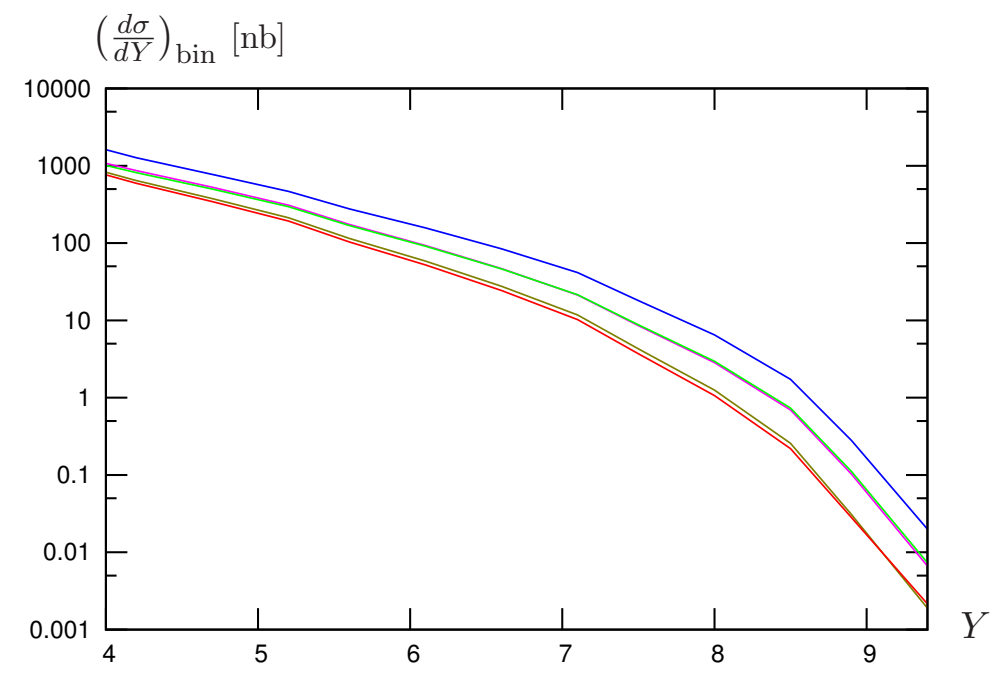

Figure 4. Differential cross-section as a function of the jet rapidity separation $Y$, integrated over bins $35 \mathrm{GeV}<\left|\mathbf{k}_{J, 1}\right|,\left|\mathbf{k}_{J, 2}\right|<60 \mathrm{GeV}$ and $0<y_{1}, y_{2}<4.7$, for the 5 scenarios described in the text, see (4.2).
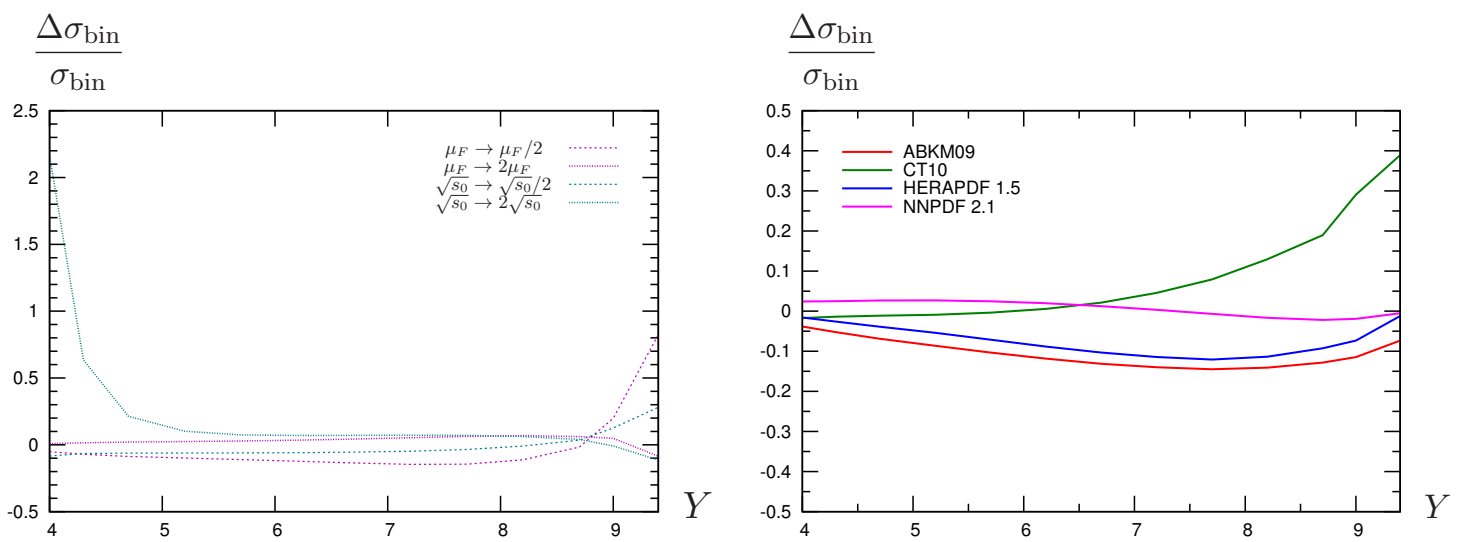

Figure 5. Left: relative variation of the cross-section when varying $\sqrt{s_{0}}$ and $\mu_{F}$ with a factor 2 . Right: relative variation of cross-section with respect to MSTW PDFs due to the replacement by other sets of PDFs, as indicated.

conformal spins $n$, we obtain very close results for the pure NLL and the collinear improved NLL approaches. This can be compared with the mixed LL jet combined with either a pure NLL Green's function or collinear improved NLL Green's function scenarios: the resulting modification is much smaller at NLL. This is in our opinion a sign of convergence of the perturbative series. One can see on figure 7 this effect already at the level of fixed $\left|\mathbf{k}_{J, 1}\right|=\left|\mathbf{k}_{J, 2}\right|=35 \mathrm{GeV}$, when passing from $n=0$ collinear improved NLL Green's function (left) to all $n$ collinear improved NLL Green's function (right). ${ }^{4}$ Note that this resummation affects only the green and the red curves in figure 7 , which are thus the only

\footnotetext{
${ }^{4}$ We plot these curves for $\sqrt{s}=7 \mathrm{TeV}$, with the same choice of parameters for $y_{i}$ as in ref. [51], i.e. $3<y_{i}<5$ and thus $6<Y<10$.
} 


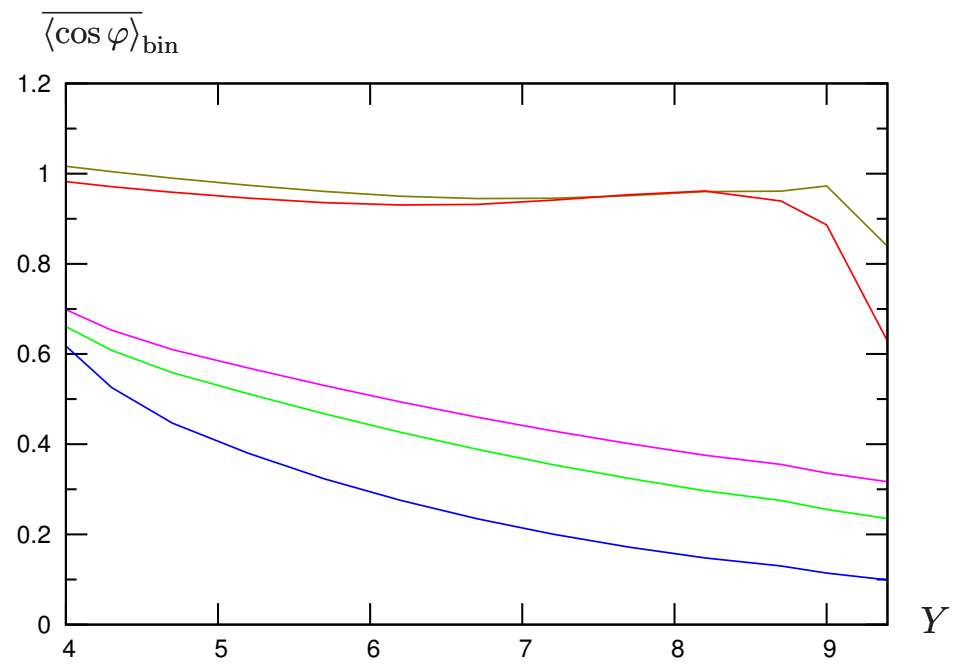

Figure 6. The bin averaged $\overline{\langle\cos \varphi\rangle}_{\text {bin }}$ as a function of the jet rapidity separation $Y$, integrated over bins $35 \mathrm{GeV}<\left|\mathbf{k}_{J, 1}\right|,\left|\mathbf{k}_{J, 2}\right|<60 \mathrm{GeV}$ and $0<y_{1}, y_{2}<4.7$, for the 5 scenarios described in the text, see (4.2).
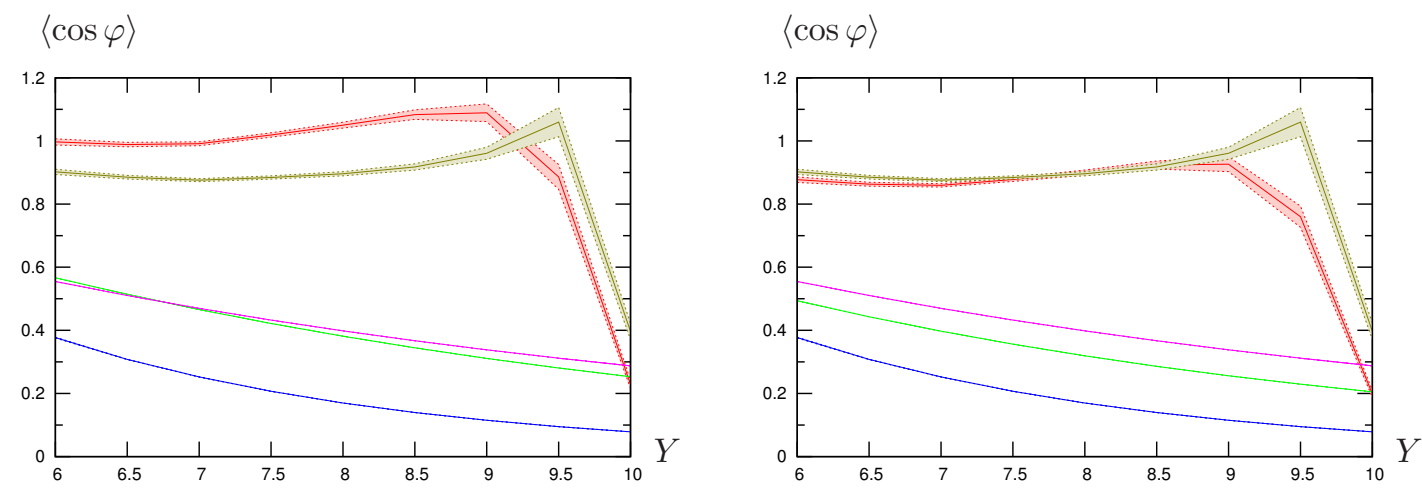

Figure 7. Left: collinear resummation for $n=0$; right: collinear resummation for all $n$.

modified ones when passing from figure 7 (L) to figure 7 (R). Furthermore, we see that including the collinear resummation for all $n$ does not lead anymore to $\langle\cos \varphi\rangle$ potentially above 1 (except in the very large $Y$ domain, due to the kinematical boundary effect).

We have made a similar check, in the three scenarios with LL jet vertices, between the results obtained with our code and the one used in ref. [50]. These results are in very good agreement, taking into account the slightly different numerical treatments and the fact that we use scheme 3 of ref. [60] while the code of ref. [50] is based on scheme 4 .

The predictions of figure 6 show that, contrarily to the natural expectation, the inclusion of mini-jets between the two tagged jets, when performed at full NLL, does not break the very high correlation between these two jets. Moreover, the obtained decorrelation effect is very flat with respect to $Y$. This is an effect which takes origin from the NLL corrections to vertices.

We now study the stability of this result with respect to changes of parameters, within the pure NLL approximation. The variation due to change of the scales $s_{0}$ and $\mu_{F}$ is shown in figure $8(\mathrm{~L})$. The effect is sizeable, but does not change the conclusion that the 

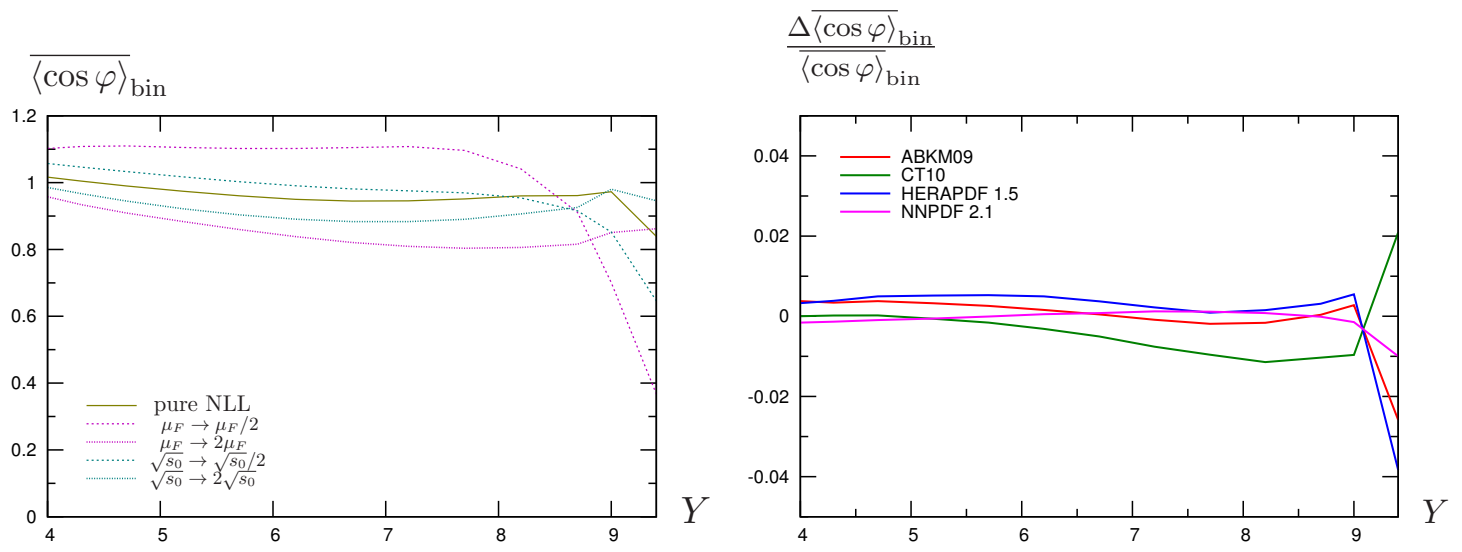

Figure 8. Left: variation of $\overline{\langle\cos \varphi\rangle}_{\text {bin }}$ when varying $\sqrt{s_{0}}$ and $\mu_{F}$ with a factor 2 . Right: relative

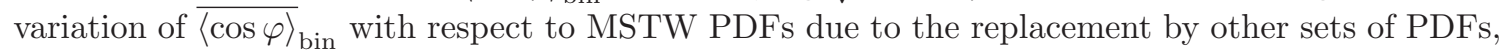
as indicated.

decorrelation remains much smaller than in the pure LL or mixed LL+NLL approaches. The second effect, due to the dependency on the set of PDFs, is shown in figure $8(R)$. This dependency is very weak, much smaller than for the cross-section, see figure $5(\mathrm{R})$. Note that this PDF dependency does not exist when using LL jet vertices.

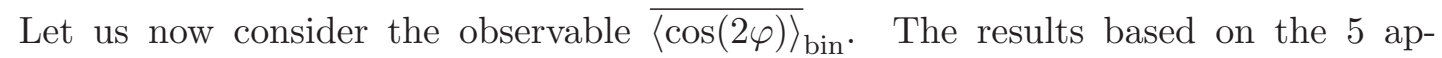
proaches (4.2) are displayed in figure $9(\mathrm{~L})$. Similar conclusions as for ${\overline{\langle\cos \varphi\rangle_{\text {bin }}}}_{\text {can be }}$ drawn. Indeed, an even more dramatic effect due to the NLL corrections to the jet vertices is observed. On the other hand, the difference between the pure NLL and the collinear improved NLL treatments is very small, this time of the same order of magnitude as the one observed between a mixed LL jet with pure NLL Green's function and the mixed LL jet with collinear improved NLL Green's function approaches.

Again, when including full NLL corrections, the decorrelation effect is rather small, and much smaller than the one obtained in non full NLL treatments, and the dependency with respect to $Y$ becomes much more flattish.

In figure $9(\mathrm{R})$, we show the dependency of our full NLL prediction with respect to changes of the scales $s_{0}$ and $\mu_{F}$. The variation due to $s_{0}$ changes is very small, almost negligible in comparison with the same dependency for $\overline{\langle\cos \varphi\rangle}_{\text {bin }}$ (see figure $8(\mathrm{~L})$ ). Besides, the dependency with respect to $\mu_{F}$ remains sizeable, although a bit smaller in absolute magnitude than in $\overline{\langle\cos \varphi\rangle}_{\text {bin }}$ (but comparable in relative magnitude).

Again, these dependencies do not change the conclusion that the decorrelation remains much smaller than in the pure LL or mixed LL+NLL approaches. The second effect, due to the dependency on the set of PDFs, is very weak, similar to the one shown in figure $8(\mathrm{R})$, and will not be displayed here.

It turns out that this remaining dependency with respect to $\mu_{F}$ is much reduced when considering the observable $\overline{\langle\cos (2 \varphi)\rangle}_{\text {bin }} / \overline{\langle\cos \varphi\rangle}_{\text {bin }}$. This observable is shown in figure 10 . In figure $10(\mathrm{~L})$ we display our prediction based on the 5 approaches $(4.2)$, while in figure $10(\mathrm{R})$ we show the $\sqrt{s_{0}}$ and $\mu_{F}$ dependency. The difference between the full NLL prediction (either collinearly improved or not) and the non-full NLL ones is sizeable for 

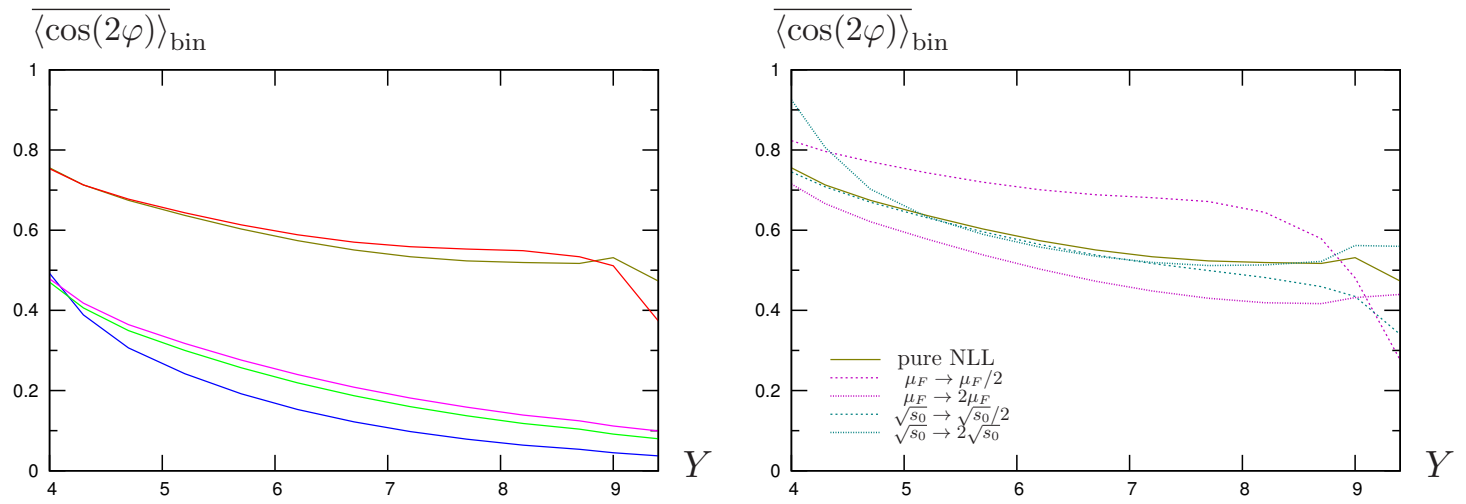

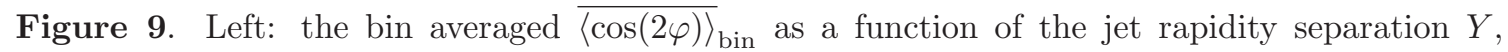
integrated over bins $35 \mathrm{GeV}<\left|\mathbf{k}_{J, 1}\right|,\left|\mathbf{k}_{J, 2}\right|<60 \mathrm{GeV}$ and $0<y_{1}, y_{2}<4.7$, for the 5 scenarios

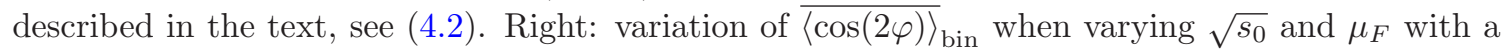
factor 2 .
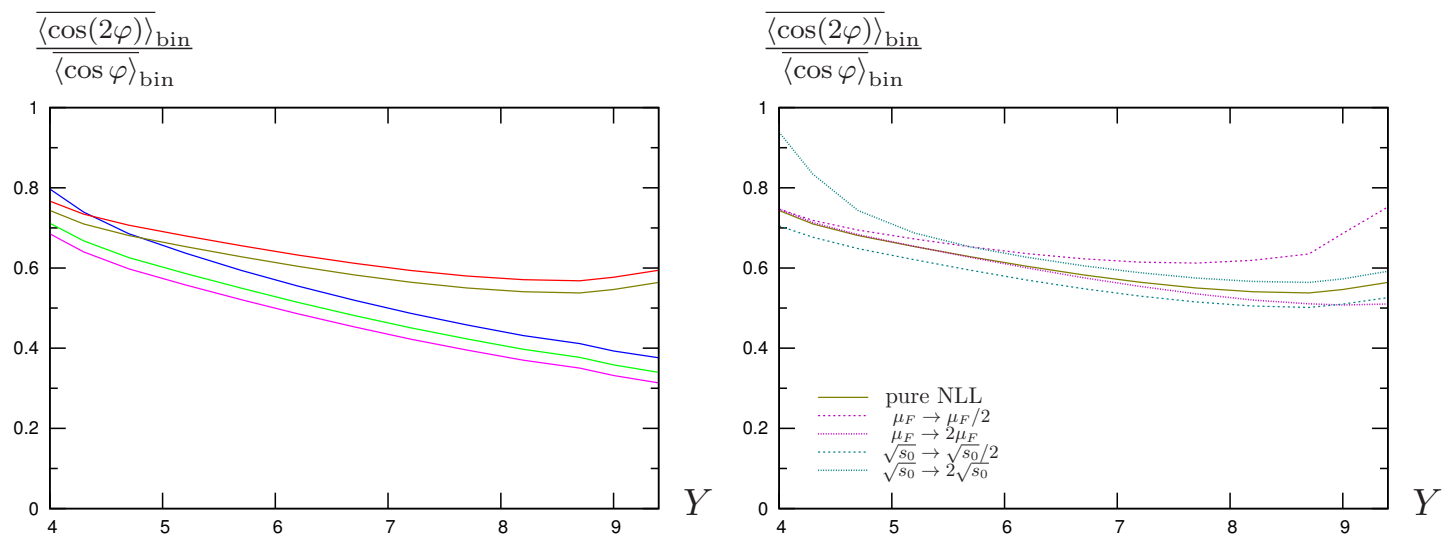

Figure 10. Left: the bin averaged $\overline{\langle\cos (2 \varphi)\rangle}_{\text {bin }}{\overline{\langle\cos \varphi\rangle}}_{\text {bin }}$ as a function of the jet rapidity separation $Y$, integrated over bins $35 \mathrm{GeV}<\left|\mathbf{k}_{J, 1}\right|,\left|\mathbf{k}_{J, 2}\right|<60 \mathrm{GeV}$ and $0<y_{1}, y_{2}<4.7$, for the 5 scenarios

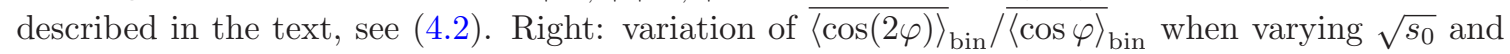
$\mu_{F}$ with a factor 2 .

$Y \gtrsim 6$, and the figure $10(\mathrm{R})$ explicitly shows that this remains valid when taking into account $\sqrt{s_{0}}$ and $\mu_{F}$ dependencies.

The extraction of higher harmonics can be as well experimentally performed. We show in figure $11(\mathrm{~L})$ our predictions for $\overline{\langle\cos (3 \varphi)\rangle}_{\text {bin }}$ based on the 5 different treatments (4.2), and the corresponding sensitivity with respect to $\sqrt{s_{0}}$ and $\mu_{F}$ in figure $11(\mathrm{R})$. In comparison with $\overline{\langle\cos (2 \varphi)\rangle}_{\text {bin }}$, again the effect of NLL corrections in jet vertices is very important, leading to a much smaller decorrelation. The dependency with respect to $s_{0}$ is similarly small, while the $\mu_{F}$ dependency is still sizeable. It is smaller in absolute magnitude than

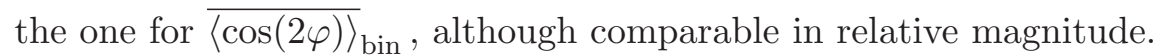

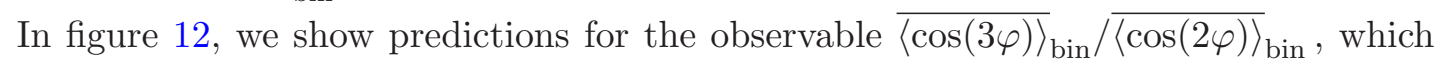
is less sensitive to changes of factorization scale $\mu_{F}$. In figure $12(\mathrm{~L})$ we see that the differences between the approaches (4.2) are not sizeable. We see in figure $12(\mathrm{R})$ that this remains true when taking into account the $\sqrt{s_{0}}$ and $\mu_{F}$ dependency. 

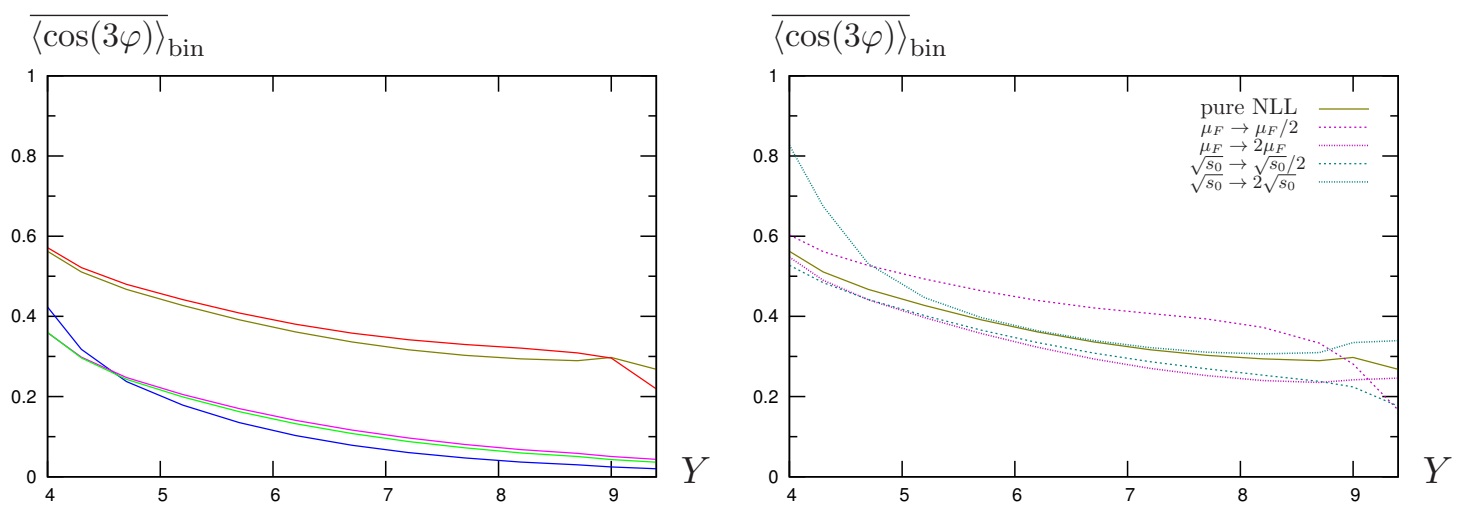

Figure 11. Left: the bin averaged $\overline{\langle\cos (3 \varphi)\rangle_{\text {bin }}}$ as a function of the jet rapidity separation $Y$, integrated over bins $35 \mathrm{GeV}<\left|\mathbf{k}_{J, 1}\right|,\left|\mathbf{k}_{J, 2}\right|<60 \mathrm{GeV}$ and $0<y_{1}, y_{2}<4.7$, for the 5 scenarios described in the text, see (4.2). Right: variation of $\overline{\langle\cos (3 \varphi)\rangle_{\text {bin }}}$ when varying $\sqrt{s_{0}}$ and $\mu_{F}$ with a factor 2 .
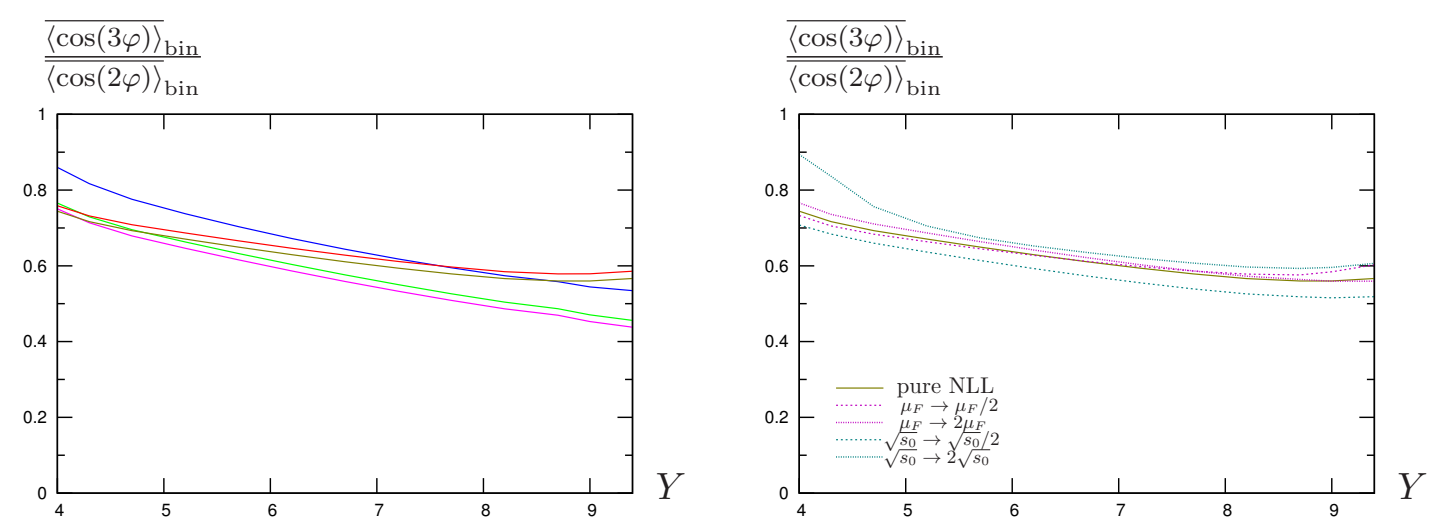

Figure 12. Left: the bin averaged ${\overline{\langle\cos (3 \varphi)\rangle_{\text {bin }}}}_{\overline{\langle\cos (2 \varphi)\rangle_{\text {bin }}}}$ as a function of the jet rapidity separation $Y$, integrated over bins $35 \mathrm{GeV}<\left|\mathbf{k}_{J, 1}\right|,\left|\mathbf{k}_{J, 2}\right|<60 \mathrm{GeV}$ and $0<y_{1}, y_{2}<4.7$, for the 5 scenarios described in the text, see (4.2). Right: variation of ${\overline{\langle\cos (3 \varphi)\rangle_{\text {bin }}}}_{\left\langle\overline{\langle\cos (2 \varphi)\rangle_{\text {bin }}}\right.}$ when varying $\sqrt{s_{0}}$ and $\mu_{F}$ with a factor 2 .

\subsection{Azimuthal distribution}

In practice, the observable which is most directly accessible in experiments is the azimuthal distribution of the two jets, defined as

$$
\frac{1}{\sigma} \frac{d \sigma}{d \varphi}=\frac{1}{2 \pi}\left\{1+2 \sum_{n=1}^{\infty} \cos (n \varphi)\langle\cos (n \varphi)\rangle\right\} .
$$

It is shown in figure 13 for LL, mixed jet LL with NLL Green's function, and mixed jet LL with collinear improved NLL Green's function, and in figure 14 for pure NLL and collinear improved NLL approaches.

The figure 13 shows that the inclusion of NLL corrections to the Green's function leads to a smaller decorrelation compared to a pure LL treatment. Comparing figure 13 with figure 14, we see that the NLL corrections to the jet vertices lead to an even larger 

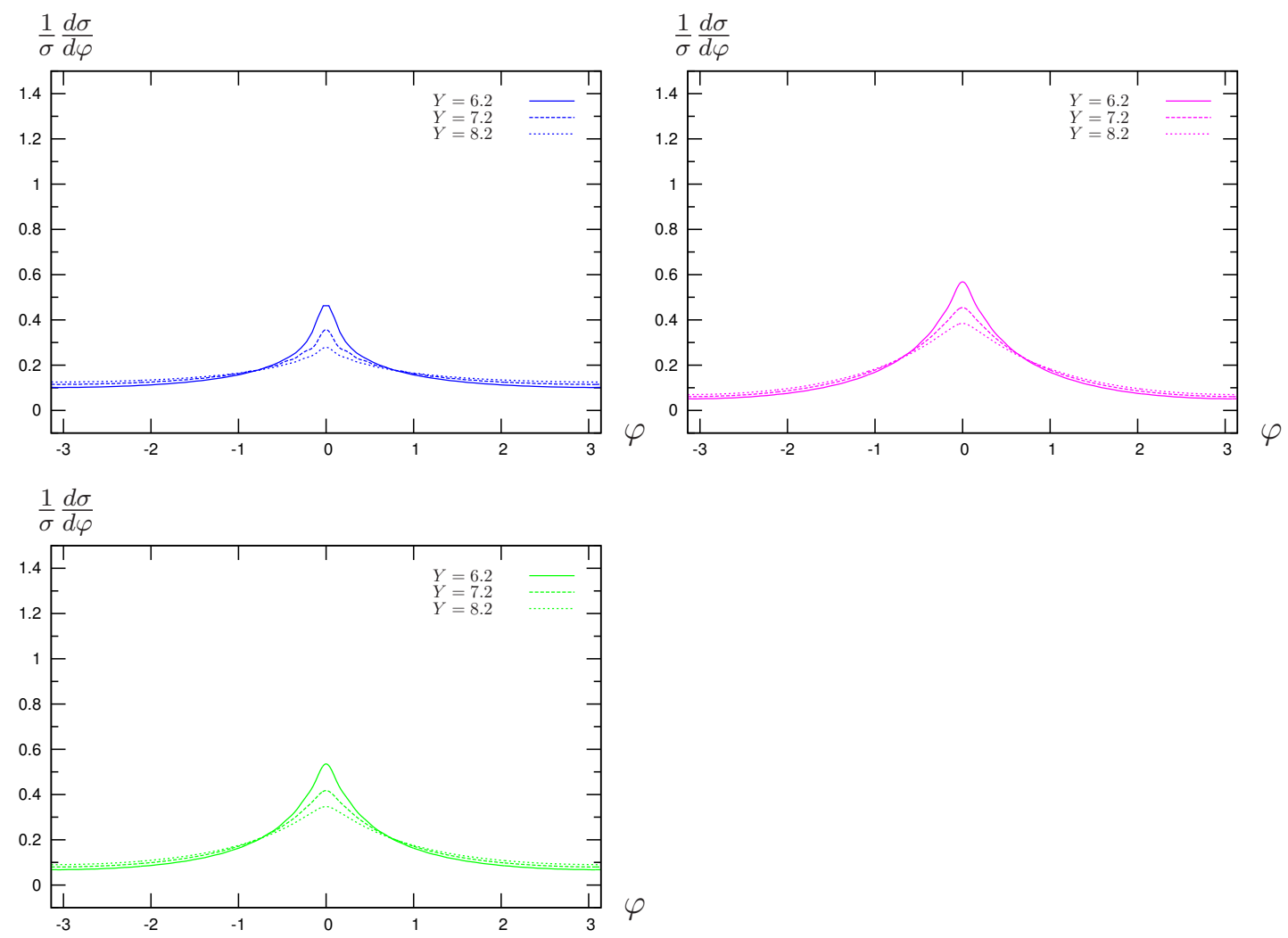

Figure 13. The azimuthal distribution $\frac{1}{\sigma} \frac{d \sigma}{d \varphi}$, for the 3 first scenarios of (4.2), for 3 values of $Y$.
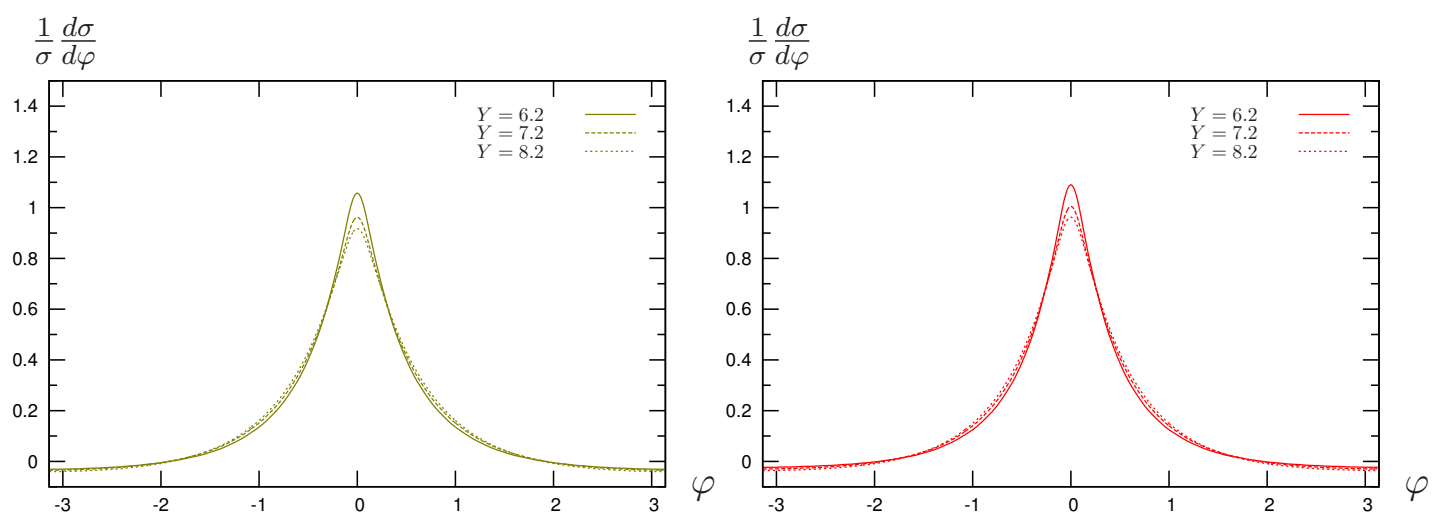

Figure 14. The azimuthal distribution $\frac{1}{\sigma} \frac{d \sigma}{d \varphi}$ for the 2 full NLL scenarios of (4.2), for 3 values of $Y$.

correlation, at fixed $Y$. When increasing $Y$, we can see on these plots that the decorrelation effect is slower, as expected in BFKL picture.

We now integrate also over $Y$ in the range $6<Y<9.4$ in addition to $\left|\mathbf{k}_{J, 1}\right|,\left|\mathbf{k}_{J, 2}\right|, y_{1}, y_{2}$. The resulting azimuthal distribution is shown in figure 15 for LL, mixed jet LL with NLL Green's function, and mixed jet LL with collinear improved NLL Green's function, and in figure 16 for the two full NLL scenarios of (4.2). In the same plots is shown the dependency with respect to $s_{0}$ and $\mu_{F}$. We see that the pure LL approach is quite dependent on the 

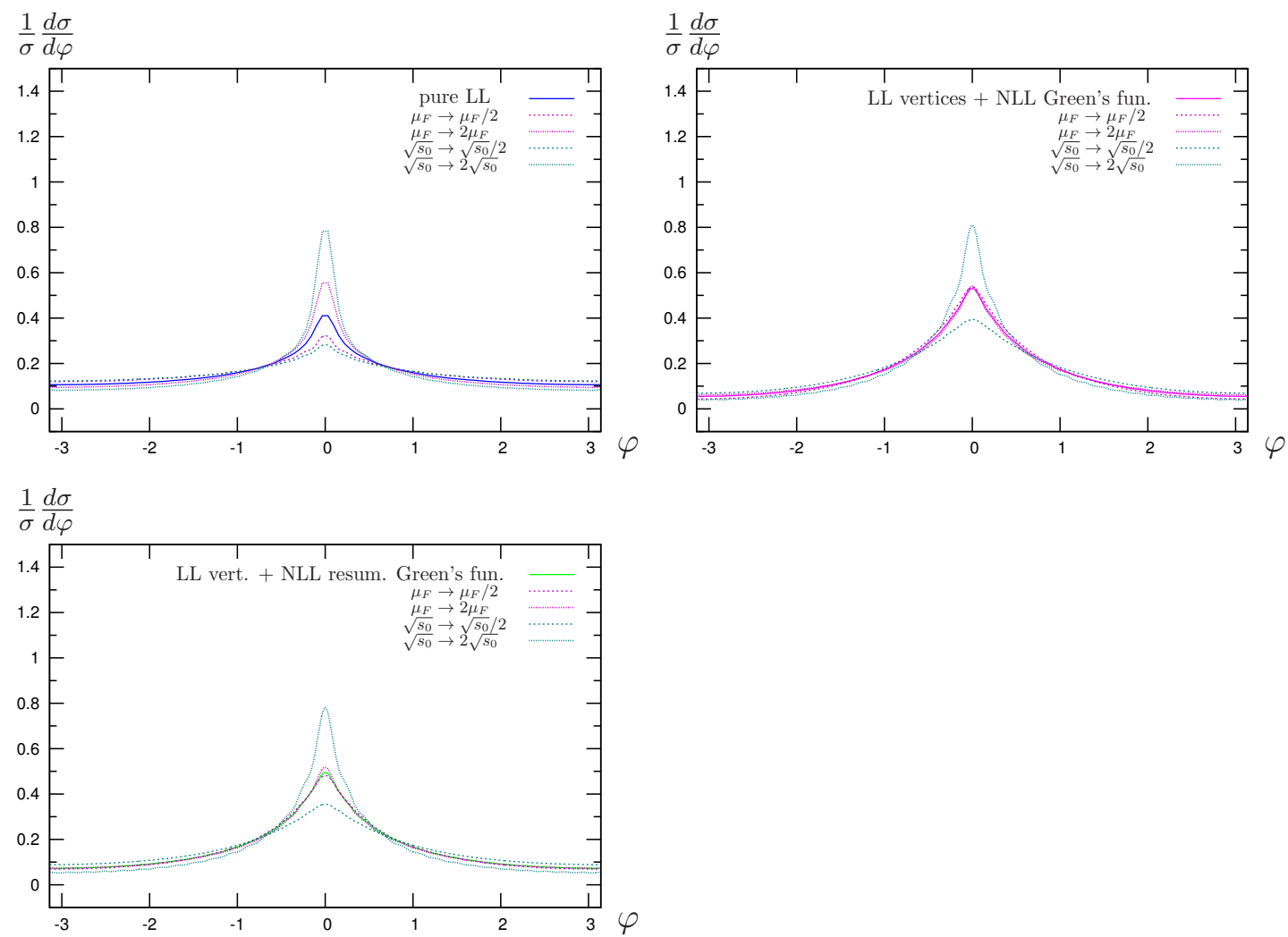

Figure 15. The azimuthal distribution $\frac{1}{\sigma} \frac{d \sigma}{d \varphi}$ integrated over bins $35 \mathrm{GeV}<\left|\mathbf{k}_{J, 1}\right|,\left|\mathbf{k}_{J, 2}\right|<60 \mathrm{GeV}$, $0<y_{1}, y_{2}<4.7$ and $6<Y<9.4$, for the 3 first scenarios of (4.2), including a variation of $\sqrt{s_{0}}$ and $\mu_{F}$ with a factor 2 with respect to the central values.
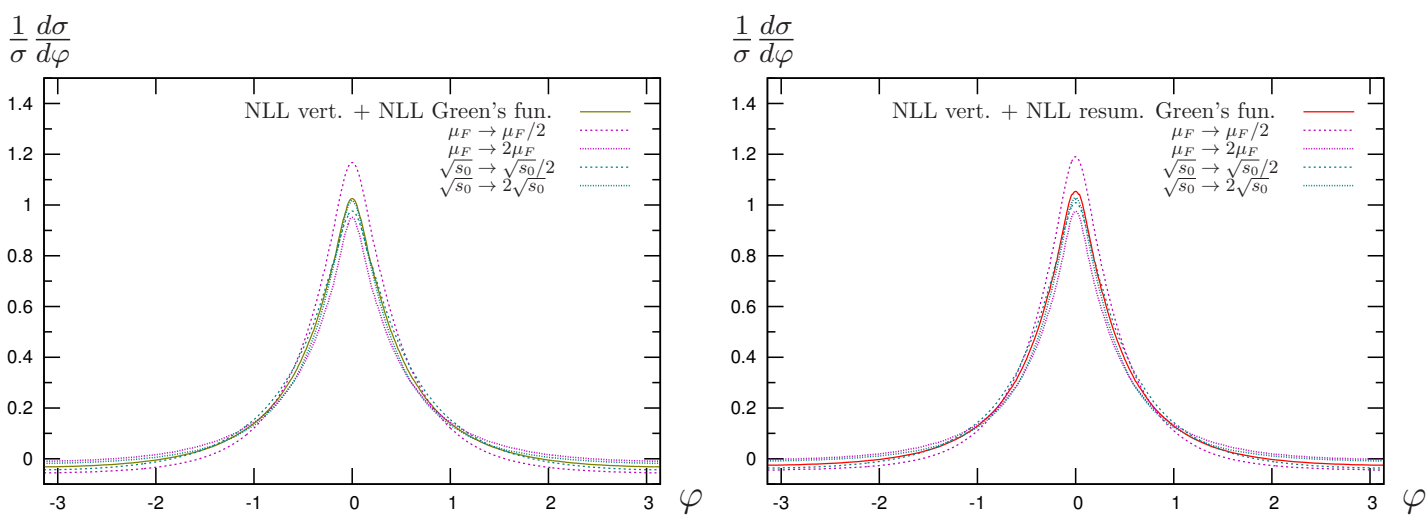

Figure 16. The azimuthal distribution $\frac{1}{\sigma} \frac{d \sigma}{d \varphi}$ integrated over bins $35 \mathrm{GeV}<\left|\mathbf{k}_{J, 1}\right|,\left|\mathbf{k}_{J, 2}\right|<60 \mathrm{GeV}$, $0<y_{1}, y_{2}<4.7$ and $6<Y<9.4$, for the 2 full NLL scenarios of (4.2), including a variation of $\sqrt{s_{0}}$ and $\mu_{F}$ with a factor 2 with respect to the central values.

scales $\sqrt{s_{0}}$ and $\mu_{F}$, whereas a mixed treatment using LL vertices with NLL Green's function shows a smaller dependency on $\mu_{F}$. The full NLL approaches are much more stable with respect to $\sqrt{s_{0}}$, while still $\mu_{F}$ dependent. 


\section{Results: asymmetric configuration}

An asymmetric configuration with very different $k_{J \min , 1}$ and $k_{J \min , 2}$ allows us to compare our predictions with the ones obtained by fixed order NLO approaches, since it is known that symmetric configurations lead to unstable predictions in fixed order calculation [65, 66]. Here we compare our predictions with the results obtained by the DiJET code [54]. Below we show the same observables as the ones which we considered in section 4 for the symmetric configuration, now supplemented by a comparison with the DIJET predictions, for which we include a scale uncertainty on $\mu_{F}$ of a factor 2 , for every plot. ${ }^{5}$ We consider bins with cuts

$$
\begin{aligned}
& 35 \mathrm{GeV}<\quad\left|\mathbf{k}_{J, 1}\right|,\left|\mathbf{k}_{J, 2}\right| \quad<60 \mathrm{GeV}, \\
& 50 \mathrm{GeV}<\operatorname{Max}\left(\left|\mathbf{k}_{J, 1}\right|,\left|\mathbf{k}_{J, 2}\right|\right), \\
& 0<\quad y_{1}, y_{2} \quad<4.7 .
\end{aligned}
$$

Since the cross-section is dominated by minimal allowed values of $\left|\mathbf{k}_{J, 1}\right|,\left|\mathbf{k}_{J, 2}\right|$, such a choice of binning reduces the domain where $\left|\mathbf{k}_{J, 1}\right|$ and $\left|\mathbf{k}_{J, 2}\right|$ are very close to each other, for which unstable results at fixed order may be a source of worry.

As the behaviour of the BFKL results is very similar to the one for a symmetric configuration, we will mainly focus in this section on the comparison with DiJET.

\subsection{Cross-section}

On the figure 17, we show the cross-section. This figure shows surprising results: the fixed order results are above BFKL predictions, contrarily to the expectation. This difference remains valid after including the effect of possible variations of the parameters $s_{0}$ and $\mu_{F}$, as can be seen in figure 18 .

\subsection{Azimuthal correlations}

We now consider the azimuthal correlation, for which we again compare predictions based on 5 kinds of BFKL scenarios with a fixed order NLO prediction. The predictions for $\overline{\langle\cos \varphi\rangle}_{\text {bin }}$ are displayed in figure 19 (L). The two full NLL BFKL predictions (pure and collinearly improved) are noticeably above the fixed order NLO prediction. However, the figure $19(\mathrm{R})$ shows that the uncertainties with respect to $s_{0}$ and $\mu_{F}$ are quite significant, and do not allow to distinguish between the full NLL BFKL predictions and the fixed order NLO one.

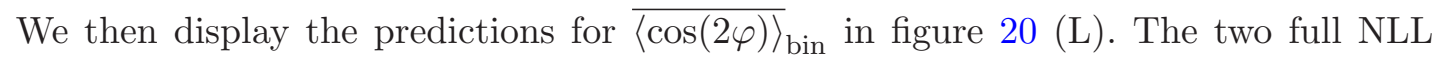
BFKL predictions (pure and collinearly improved) are now a bit below the fixed order NLO prediction, and again, the figure $20(\mathrm{R})$ shows that the uncertainties with respect to $s_{0}$ and $\mu_{F}$ are quite significant, and do not allow to distinguish between the full NLL BFKL predictions and the fixed order NLO one with this observable.

\footnotetext{
${ }^{5}$ Note that the results obtained with DiJet use a scale $\mu_{F}=\frac{\left|\mathbf{k}_{J, 1}\right|+\left|\mathbf{k}_{J, 2}\right|}{2}$, which is very close in the domain we consider to the value $\sqrt{\left|\mathbf{k}_{J, 1}\right| \cdot\left|\mathbf{k}_{J, 2}\right|}$ we use in our BFKL calculation
} 


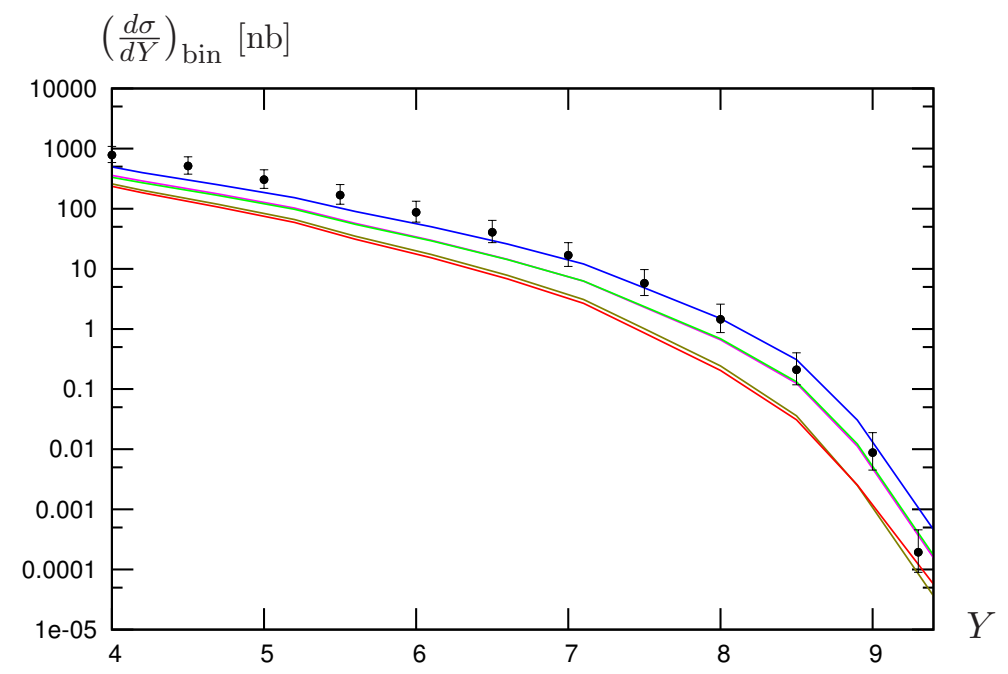

Figure 17. Differential cross-section as a function of the jet rapidity separation $Y$, using cuts defined in (5.1). The different curves correspond to the 5 scenarios (4.2). The dots correspond to the predictions of the DIJET code.

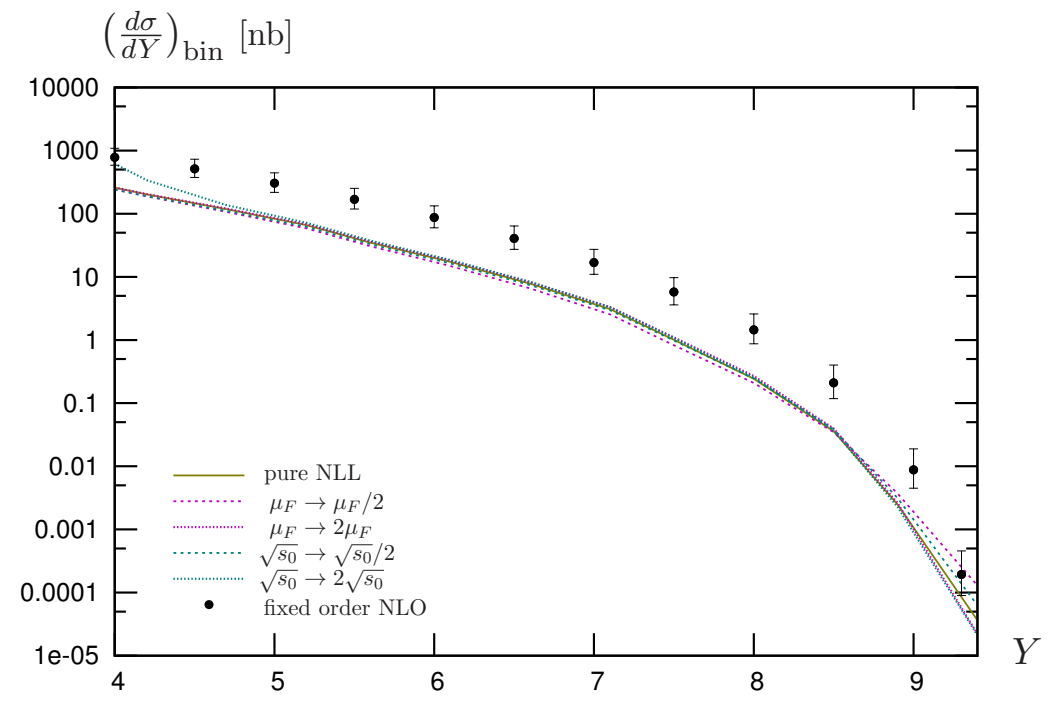

Figure 18. Differential cross-section as a function of the jet rapidity separation $Y$, using cuts defined in (5.1), in the full NLL approximation. The dots correspond to the predictions of the DIJET code. We show the effect of a variation of $\sqrt{s_{0}}$ and $\mu_{F}$ with a factor 2 with respect to the central values.

Let us now study the observable $\frac{\overline{\langle\cos (2 \varphi)\rangle_{\text {bin }}}}{\overline{\langle\cos \varphi\rangle_{\text {bin }}}}$. The fact that in the full NLL BFKL

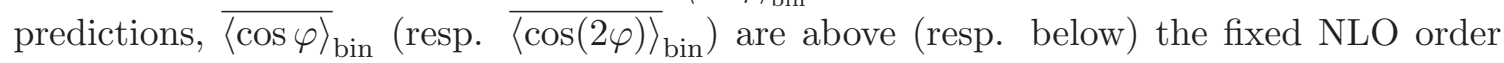
predictions now leads to a ratio which is very significantly, in the full NLL BFKL approximation, under the NLO fixed order one, as can be seen from figure 21 (L). This difference is not washed out when including the uncertainties due to $s_{0}$ and $\mu_{F}$ variations, as shown 

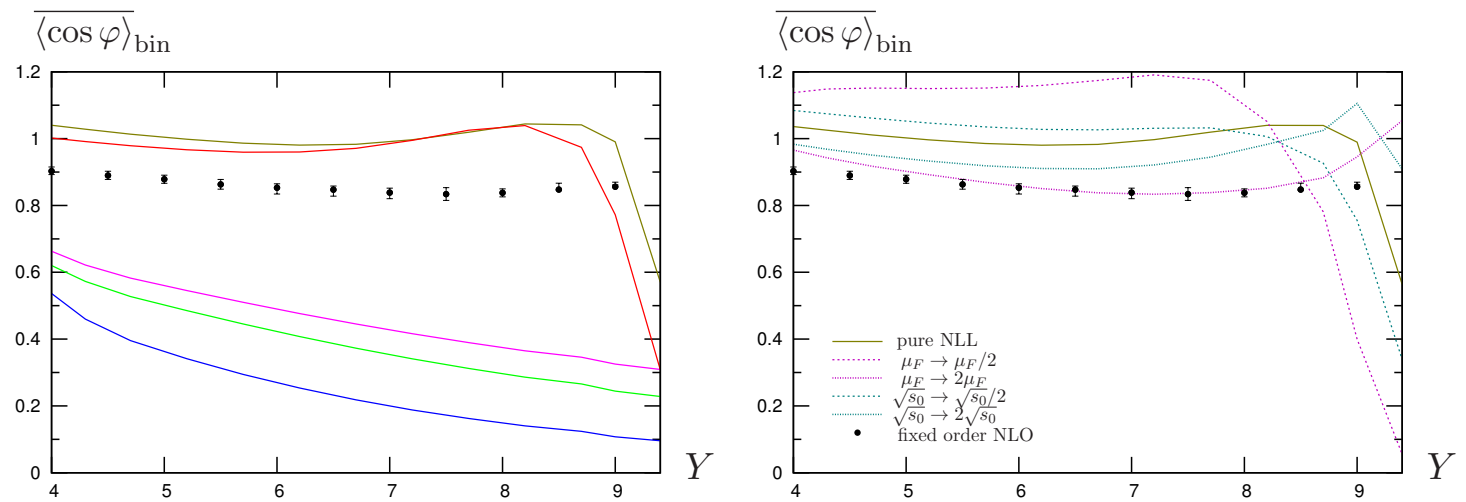

Figure 19. Left: the bin averaged $\overline{\langle\cos \varphi\rangle}_{\text {bin }}$ as a function of the jet rapidity separation $Y$, using cuts defined in (5.1), for the 5 scenarios described in the text, see (4.2). Right: variation of $\overline{\langle\cos \varphi\rangle}_{\text {bin }}$ when varying $\sqrt{s_{0}}$ and $\mu_{F}$ with a factor 2 . The dots correspond to the predictions of the DiJET code.
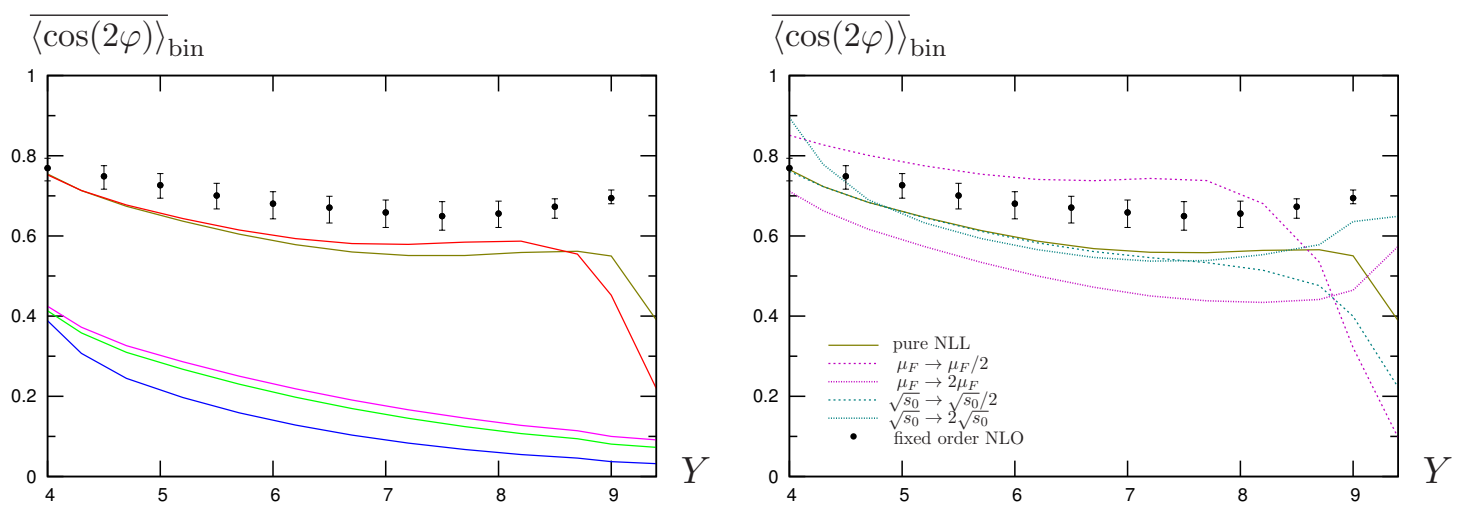

Figure 20. Left: the bin averaged $\overline{\langle\cos (2 \varphi)\rangle}_{\text {bin }}$ as a function of the jet rapidity separation $Y$, using cuts defined in (5.1), for the 5 scenarios described in the text, see (4.2). Right: variation of

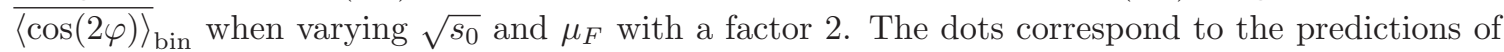
the DIJET code.

in figure $21(\mathrm{R})$. We want to emphasize the fact that this is valid in particular in the region $Y \sim 6$, for which according to the discussion of section 3.2, the corrections due to energy-momentum conservation are not expected to be very significant.

We finally consider the observable $\overline{\langle\cos (3 \varphi)\rangle}$ bin in figure 22 (L). The two full NLL BFKL predictions (pure and collinearly improved) are now significantly below the fixed order NLO prediction. The figure $22(\mathrm{R})$ shows that the uncertainties with respect to $s_{0}$ and $\mu_{F}$ are quite significant, and can marginally alter the possibility of distinguishing the two types of scenarios, mainly due to the $\mu_{F}$ uncertainty. Considering the ratio $\frac{{\overline{\langle\cos (3 \varphi)\rangle_{\text {bin }}}}_{\langle\cos (2 \varphi)\rangle_{\text {bin }}}}{\text {, }}$ we observe that this latter observable is much more favorable, as can be seen from figure 23 .

We do not study here the azimuthal distribution as we have done in the symmetric case of section 4.3. Indeed it is not possible to confront our BFKL predictions with the fixed order NLO predictions of the DIJET code, due to instabilities when evaluating the higher harmonics, which are necessary for a precise study of $\varphi$ distribution, using the DiJeT code. 

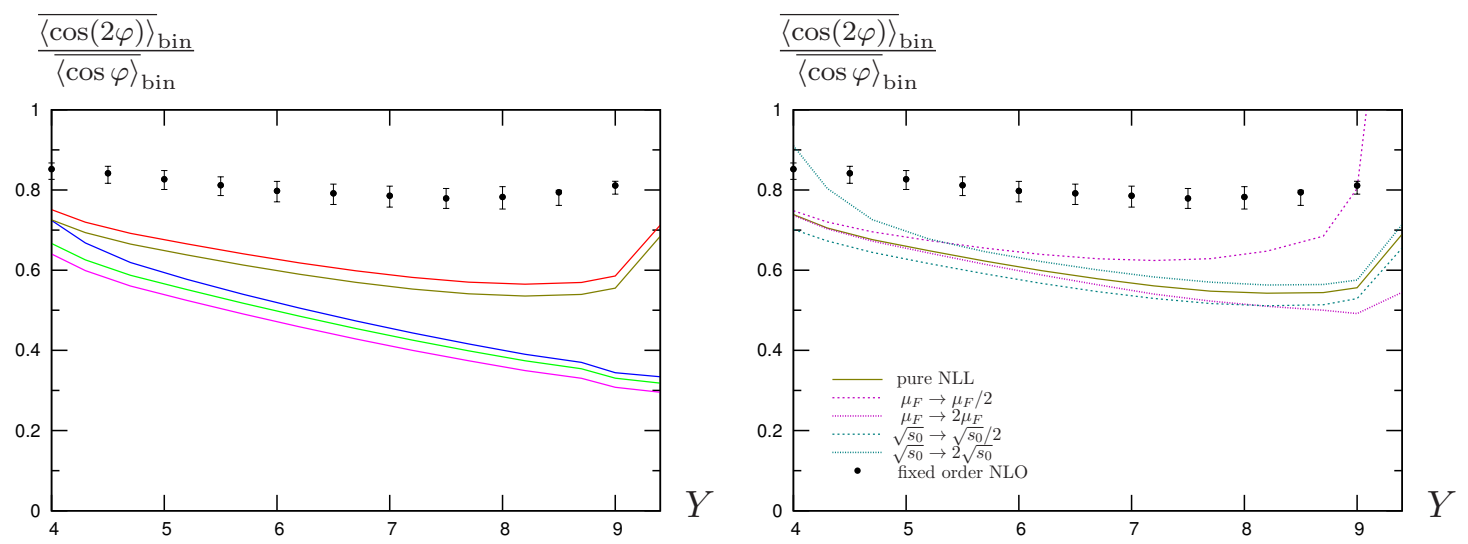

Figure 21. Left: the bin averaged $\overline{\langle\cos (2 \varphi)\rangle}_{\text {bin }} / \overline{\langle\cos \varphi\rangle}_{\text {bin }}$ as a function of the jet rapidity separation $Y$, using cuts defined in (5.1), for the 5 scenarios described in the text, see (4.2). Right: variation

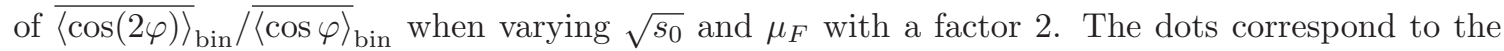
predictions of the DIJET code.
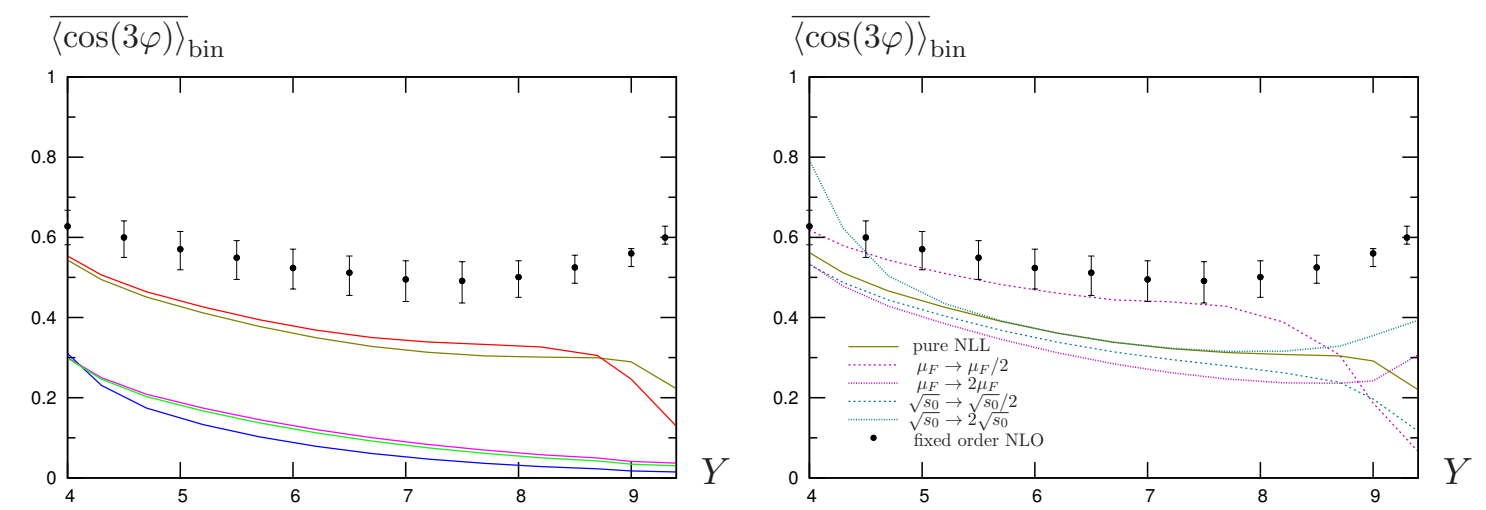

Figure 22. Left: the bin averaged $\overline{\langle\cos (3 \varphi)\rangle}$ bin as a function of the jet rapidity separation $Y$, using cuts defined in (5.1), for the 5 scenarios described in the text, see (4.2). Right: variation of

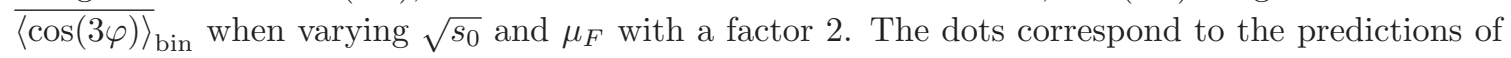
the DIJET code.

\section{Limit of small-R cone}

A detailed study, based on the work of ref. [67] where the jet vertices were computed in an approximated small $R$ treatment, shows that the difference between an exact treatment, as used in the present work, and that small $R$ approximation is small. This is illustrated in figure 24 for $R=0.3$ and in figure 25 for $R=0.5$, and shows explicitly the consistency of the two approaches. This small $R$ limit has been used in ref. [68] for phenomenological studies. ${ }^{6}$ In this paper, it is stated that sizeable differences are obtained when comparing with the results we got in ref. [51], with the same set of parameters. We believe that this is mainly due to the way NNLL corrections, which are beyond the precision of both studies,

\footnotetext{
${ }^{6}$ We thank the authors of ref. [68] for pointing out that the values for $\mathcal{C}_{1}$ and $\mathcal{C}_{2}$ given in ref. [51] should be multiplied by a factor of 2 to get the proper normalization.
} 

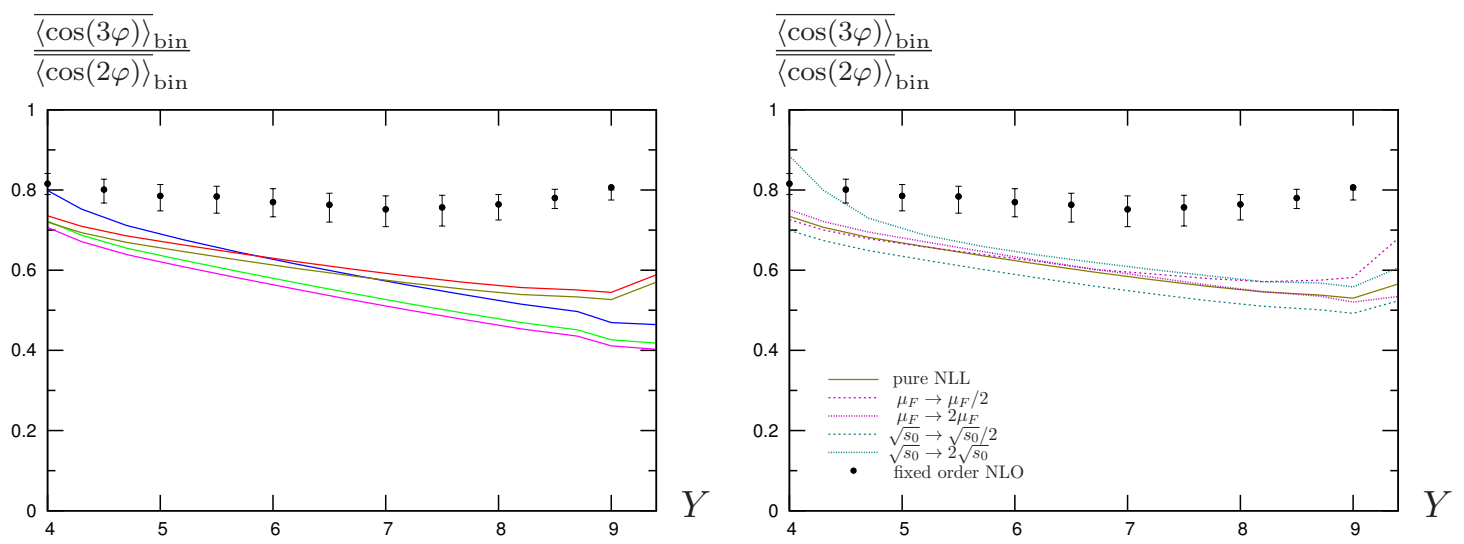

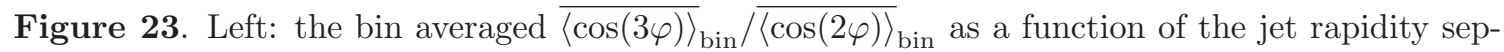
aration $Y$, using cuts defined in (5.1), for the 5 scenarios described in the text, see (4.2). Right: variation of $\overline{\langle\cos (3 \varphi)\rangle_{\text {bin }}} / \overline{\langle\cos (2 \varphi)\rangle_{\text {bin }}}$ when varying $\sqrt{s_{0}}$ and $\mu_{F}$ with a factor 2 . The dots correspond to the predictions of the DiJET code.
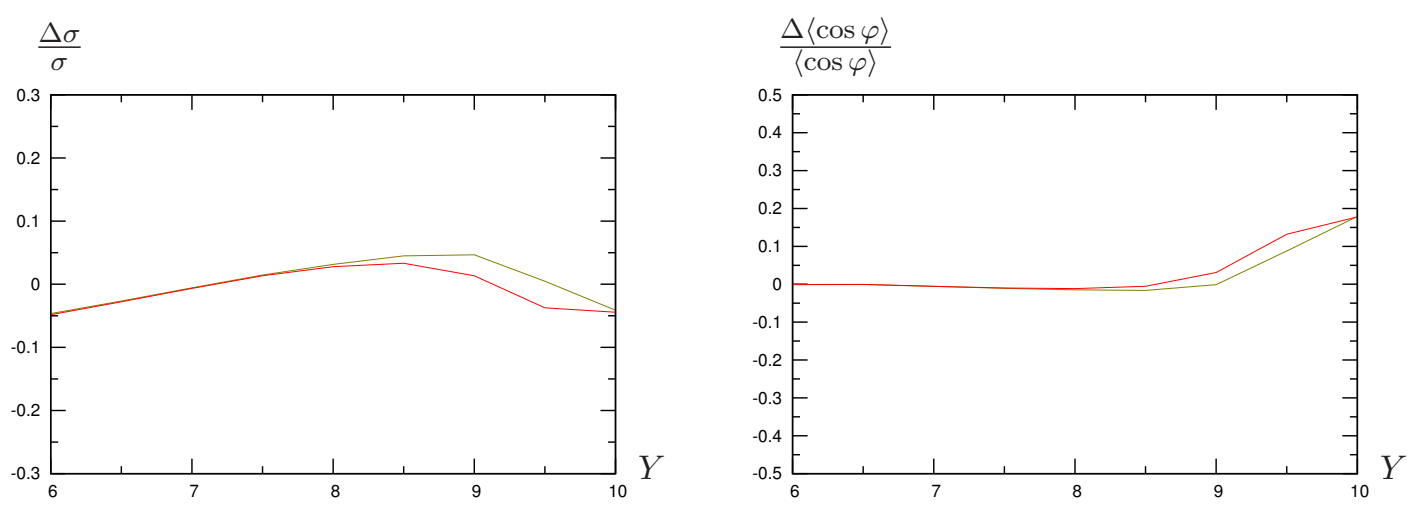

Figure 24. Relative difference between an exact treatment of the cone size and the small cone approximation for $R=0.3$ and $\left|\mathbf{k}_{J, 1}\right|=\left|\mathbf{k}_{J, 2}\right|=35 \mathrm{GeV}$, in the two full NLL BFKL scenarios.

are treated. Indeed, when convoluting jet vertices with the Green's function, there is a freedom to neglect terms of magnitude $\alpha_{s}^{3} Y$. A close inspection on the way both papers deal with such contributions shows that in ref. [68], eq. (42), terms involving the product of NLL corrections in both vertices are explicitly neglected, while they are kept in ref. [51] and in the present study.

\section{Conclusions}

In this paper, we have continued our NLL analysis of Mueller-Navelet jets, at the partonic level, concentrating on the kinematical conditions of ongoing experiments ATLAS and CMS at LHC. We have made a detailed study of the azimuthal distributions for the first time at full NLL BFKL accuracy. Finally, we confronted our predictions with the fixed order predictions based on the DiJET code. In this regard, note that our analysis, like the one based on the DIJET code, does not take into account hadronization effects. 

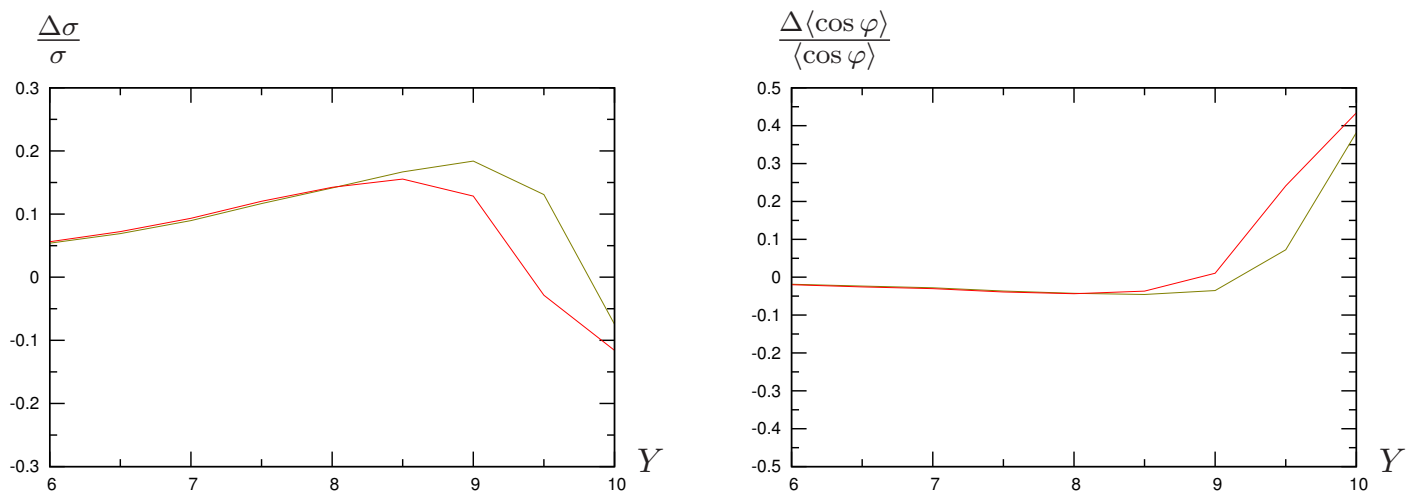

Figure 25. Relative difference between an exact treatment of the cone size and the small cone approximation for $R=0.5$ and $\left|\mathbf{k}_{J, 1}\right|=\left|\mathbf{k}_{J, 2}\right|=35 \mathrm{GeV}$, in the two full NLL BFKL scenarios.

The present analysis is based on the original assumption of Mueller and Navelet that the two tagged jets are the most forward and most backward ones. However, it turns out that extracting such exclusive events is not an easy task experimentally, and that the real measurements may require to be more inclusive, with some possible activity between a given proton and the corresponding jet with a small relative rapidity. This effect was investigated at LL BFKL order [69, 70]. A similar study with full NLL BFKL accuracy could be of phenomenological interest, and is left for further study.

We did not estimate the importance of potentially competing production mechanisms involving multiparton interactions (MPIs). These are relevant for processes involving final states with fixed and moderate values of transverse momentum [71], leading to unsuppressed contribution from the twist counting point of view. In the context of the present study, the values of $\mathbf{k}_{i}$ are in principle large enough to kill such a mechanism. However, the resummation of high energy effects may compensate this twist suppression [72, 73], and the evaluation of their importance is left for future analysis.

We would like to comment here on the fact that the question of evaluating the effect of high energy resummation, which is at the heart of the present study, here with full NLL BFKL accuracy, and of the potential importance of MPIs type of contributions, may also be addressed in the context of di-hadron correlations (with a large rapidity separation between them) in high multiplicity events in proton-proton as well as proton-ion or ion-ion collisions. The measurement of the azimuthal distribution of a hadron pair, which has been performed at RHIC and LHC [74-86], reveals two dominant configurations, one which is nearside (the so-called "ridge" effect), and one which is awayside. The first one may be understood as a sign of saturation (e.g. by means of the Color Glass Condensate approach [87-90]), which mechanism is based on the fact that in the high density regime, the scattering is dominated by two decorrelated chains of gluons which radiate, therefore leading to a small relative azimuthal angle between the two hadrons. This picture is somehow similar to the one behind MPIs, here in the context of saturated sources. On the other hand, the understanding of the second peak, with two almost back-to-back hadrons, may be improved by including the effect of multiple gluon radiation similarly to the situation encountered in the Mueller Navelet jets (although the $\mathbf{k}_{i}$ are here much larger than the 
ones considered in the di-hadron correlation studies), in which a single chain is responsible for the radiation [91-93]. In the spirit of the present study, the inclusion of NLL corrections may modify the azimuthal distribution for this awayside region.

The predictions of the present study confirm the main result of ref. [51] that the effect of NLL corrections to jets vertices is dramatically large, similar in magnitude to the one due to the NLL Green's function corrections. In particular, we observe a very important change for the observables $\langle\cos (n \varphi)\rangle$ when passing from LL to NLL vertices. This puzzling effect is not easy to understand analytically due to the complicated structure of the NLL vertices and is left for further studies.

We have investigated the stability of our predictions with respect to changes of factorization scale $\mu_{F}$, of scale $s_{0}$ and of sets of PDFs. For the cross-section, in comparison with scenarios using LL jet vertices, the predictions are much more stable with respect to variation of $\mu_{F}$ and $s_{0}$, and of similar small order of magnitude for PDFs variations. Our full NLL BFKL predictions are surprisingly sizeably below the fixed order NLO prediction.

For the decorrelation effect, the full NLL BFKL predictions and fixed order NLO one are very close for $\langle\cos \varphi\rangle$ and $\langle\cos 2 \varphi\rangle$. They are very flat in rapidity $Y$, but still rather dependent on $s_{0}$, and specially on $\mu_{F}$, while weakly dependent on PDFs.

We have taken into account the effect of collinearly improved NLL BFKL Green's function. Including these effects for non zero conformal spins $n$ has an important impact on our predictions for azimuthal decorrelation. It leads to results which are very close to the pure NLL BFKL treatment.

The angular $\varphi$ distribution which we predict at full NLL BFKL is very strongly peaked around 0 and does not evolve strongly with respect to $Y$. This prediction significantly differs from the ones based on LL jet vertices, and is stable when changing $\mu_{F}, s_{0}$ and PDFs.

Finally, we have shown that for the ratios $\langle\cos 2 \varphi\rangle /\langle\cos \varphi\rangle$ and $\langle\cos 3 \varphi\rangle /\langle\cos 2 \varphi\rangle$ the differences between NLL BFKL and fixed order NLO are sizeable, and stable with respect to scale choices.

To conclude, our analysis suggests that the ratios of harmonics are most suitable observables to distinguish between full NLL BFKL predictions and fixed order NLO ones.

\section{Acknowledgments}

We acknowledge the collaboration with Florian Schwennsen and Dimitri Colferai, which was the starting basis of the present study.

We are very grateful to Michel Fontannaz, Cyrille Marquet and Christophe Royon for providing their codes and for stimulating discussions.

We strongly thank Grzegorz Brona, David d'Enterria, Hannes Jung, Victor Kim and Maciej Misiura for many discussions and fruitful suggestions on the experimental aspects of this study.

S. W. thanks the participants of MPI@TAU for discussions, and Halina Abramowicz for support. L. S. and S. W. thank the participants of the CMS small- $x$ and forward physics working group for discussions, and G. Salam for support. 
This work is partly supported by the French-Polish collaboration agreement Polonium, the Polish Grant NCN No. DEC-2011/01/B/ST2/03915, the P2IO Labex and the Joint Research Activity Study of Strongly Interacting Matter (acronym HadronPhysics3, Grant Agreement n.283286) under the Seventh Framework Programme of the European Community.

Open Access. This article is distributed under the terms of the Creative Commons Attribution License which permits any use, distribution and reproduction in any medium, provided the original author(s) and source are credited.

\section{References}

[1] V.S. Fadin, E. Kuraev and L. Lipatov, On the Pomeranchuk singularity in asymptotically free theories, Phys. Lett. B 60 (1975) 50 [InSPIRE].

[2] E. Kuraev, L. Lipatov and V.S. Fadin, Multi-Reggeon processes in the Yang-Mills theory, Sov. Phys. JETP 44 (1976) 443 [Zh. Eksp. Teor. Fiz. 71 (1976) 840] [INSPIRE].

[3] E. Kuraev, L. Lipatov and V.S. Fadin, The Pomeranchuk singularity in non-Abelian gauge theories, Sov. Phys. JETP 45 (1977) 199 [Zh. Eksp. Teor. Fiz. 72 (1977) 377] [InSPIRE].

[4] I. Balitsky and L. Lipatov, The Pomeranchuk singularity in quantum chromodynamics, Sov. J. Nucl. Phys. 28 (1978) 822 [Yad. Fiz. 28 (1978) 1597] [inSPIRE].

[5] V. Gribov and L. Lipatov, Deep inelastic ep scattering in perturbation theory, Sov. J. Nucl. Phys. 15 (1972) 438 [Yad. Fiz. 15 (1972) 781] [InSPIRE].

[6] L. Lipatov, The parton model and perturbation theory, Sov. J. Nucl. Phys. 20 (1975) 94 [Yad. Fiz. 20 (1974) 181] [INSPIRE].

[7] G. Altarelli and G. Parisi, Asymptotic freedom in parton language, Nucl. Phys. B 126 (1977) 298 [inSPIRE].

[8] Y.L. Dokshitzer, Calculation of the structure functions for deep inelastic scattering and $e^{+} e^{-}$ annihilation by perturbation theory in quantum chromodynamics., Sov. Phys. JETP 46 (1977) 641 [Zh. Eksp. Teor. Fiz. 73 (1977) 1216] [InSPIRE].

[9] A. Askew, K.J. Golec-Biernat, J. Kwiecinski, A.D. Martin and P. Sutton, Implications of scaling violations of $F_{2}$ at HERA for perturbative QCD, Phys. Lett. B 325 (1994) 212 [hep-ph/9311376] [INSPIRE].

[10] H. Navelet, R.B. Peschanski, C. Royon and S. Wallon, Proton structure functions in the dipole picture of BFKL dynamics, Phys. Lett. B 385 (1996) 357 [hep-ph/9605389] [INSPIRE].

[11] S. Munier and R.B. Peschanski, High-energy factorization predictions for the charm structure function $F_{2}^{c}$ at HERA, Nucl. Phys. B 524 (1998) 377 [hep-ph/9802230] [INSPIRE].

[12] J. Bartels, A. De Roeck and H. Lotter, The $\gamma^{*} \gamma^{*}$ total cross-section and the BFKL Pomeron at $e^{+} e^{-}$colliders, Phys. Lett. B 389 (1996) 742 [hep-ph/9608401] [INSPIRE].

[13] S. Brodsky, F. Hautmann and D. Soper, Probing the QCD Pomeron in $e^{+} e^{-}$collisions, Phys. Rev. Lett. 78 (1997) 803 [Erratum ibid. 79 (1997) 3544] [hep-ph/9610260] [INSPIRE].

[14] A. Bialas, W. Czyz and W. Florkowski, Total $\gamma^{*} \gamma^{*}$ cross-section and the BFKL Pomeron, Eur. Phys. J. C 2 (1998) 683 [hep-ph/9705470] [INSPIRE]. 
[15] M. Boonekamp, A. De Roeck, C. Royon and S. Wallon, $\gamma^{*} \gamma^{*}$ total cross-section in the dipole picture of BFKL dynamics, Nucl. Phys. B 555 (1999) 540 [hep-ph/9812523] [INSPIRE].

[16] J. Kwiecinski and L. Motyka, Probing the QCD Pomeron in doubly tagged $e^{+} e^{-}$collisions, Phys. Lett. B 462 (1999) 203 [hep-ph/9905567] [INSPIRE].

[17] S.J. Brodsky, V.S. Fadin, V.T. Kim, L.N. Lipatov and G.B. Pivovarov, The QCD Pomeron with optimal renormalization, JETP Lett. 70 (1999) 155 [hep-ph/9901229] [INSPIRE].

[18] S.J. Brodsky, V.S. Fadin, V.T. Kim, L.N. Lipatov and G.B. Pivovarov, High-energy QCD asymptotics of photon-photon collisions, JETP Lett. 76 (2002) 249 [Pisma Zh. Eksp. Teor. Fiz. 76 (2002) 306] [hep-ph/0207297] [INSPIRE].

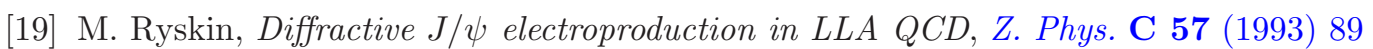
[INSPIRE].

[20] L. Frankfurt, W. Koepf and M. Strikman, Diffractive heavy quarkonium photoproduction and electroproduction in QCD, Phys. Rev. D 57 (1998) 512 [hep-ph/9702216] [INSPIRE].

[21] R. Enberg, L. Motyka and G. Poludniowski, Diffractive heavy vector meson production from the BFKL equation, Eur. Phys. J. C 26 (2002) 219 [hep-ph/0207027] [INSPIRE].

[22] D.Y. Ivanov, A. Schafer, L. Szymanowski and G. Krasnikov, Exclusive photoproduction of a heavy vector meson in QCD, Eur. Phys. J. C 34 (2004) 297 [hep-ph/0401131] [INSPIRE].

[23] D.Y. Ivanov, R. Kirschner, A. Schafer and L. Szymanowski, The light vector meson photoproduction at large t, Phys. Lett. B 478 (2000) 101 [Erratum ibid. B 498 (2001) 295] [hep-ph/0001255] [INSPIRE].

[24] R. Enberg, J.R. Forshaw, L. Motyka and G. Poludniowski, Vector meson photoproduction from the BFKL equation. 1. Theory, JHEP 09 (2003) 008 [hep-ph/0306232] [INSPIRE].

[25] G. Poludniowski, R. Enberg, J.R. Forshaw and L. Motyka, Vector meson photoproduction from the BFKL equation. 2. Phenomenology, JHEP 12 (2003) 002 [hep-ph/0311017] [INSPIRE].

[26] B. Pire, L. Szymanowski and S. Wallon, Double diffractive $\rho$-production in $\gamma^{*} \gamma^{*}$ collisions, Eur. Phys. J. C 44 (2005) 545 [hep-ph/0507038] [inSPIRE].

[27] R. Enberg, B. Pire, L. Szymanowski and S. Wallon, BFKL resummation effects in $\gamma^{*} \gamma^{*} \rightarrow \rho \rho$, Eur. Phys. J. C 45 (2006) 759 [Erratum ibid. C 51 (2007) 1015] [hep-ph/0508134] [INSPIRE].

[28] M. Segond, L. Szymanowski and S. Wallon, Diffractive production of two $\rho_{L}^{0}$ mesons in $e^{+} e^{-}$ collisions, Eur. Phys. J. C 52 (2007) 93 [hep-ph/0703166] [inSPIRE].

[29] D.Y. Ivanov and A. Papa, Electroproduction of two light vector mesons in the next-to-leading approximation, Nucl. Phys. B 732 (2006) 183 [hep-ph/0508162] [INSPIRE].

[30] D.Y. Ivanov and A. Papa, Electroproduction of two light vector mesons in next-to-leading BFKL: study of systematic effects, Eur. Phys. J. C 49 (2007) 947 [hep-ph/0610042] [INSPIRE].

[31] F. Caporale, A. Papa and A. Sabio Vera, Collinear improvement of the BFKL kernel in the electroproduction of two light vector mesons, Acta Phys. Polon. B 39 (2008) 2571 [arXiv: 0807.0525] [INSPIRE].

[32] B. Cox, J.R. Forshaw and L. Lönnblad, Hard color singlet exchange at the Tevatron, JHEP 10 (1999) 023 [hep-ph/9908464] [INSPIRE]. 
[33] R. Enberg, G. Ingelman and L. Motyka, Hard color singlet exchange and gaps between jets at the Tevatron, Phys. Lett. B 524 (2002) 273 [hep-ph/0111090] [INSPIRE].

[34] F. Chevallier, O. Kepka, C. Marquet and C. Royon, Gaps between jets at hadron colliders in the next-to-leading BFKL framework, Phys. Rev. D 79 (2009) 094019 [arXiv:0903.4598] [INSPIRE].

[35] D. Ostrovsky, NLO correction to one particle inclusive production at high-energies, Phys. Rev. D 62 (2000) 054028 [hep-ph/9912258] [InSPIRE].

[36] J. Bartels, A. Sabio Vera and F. Schwennsen, NLO inclusive jet production in $k_{T}$-factorization, JHEP 11 (2006) 051 [hep-ph/0608154] [INSPIRE].

[37] A.H. Mueller and H. Navelet, An inclusive minijet cross-section and the bare Pomeron in QCD, Nucl. Phys. B 282 (1987) 727 [INSPIRE].

[38] M. Ciafaloni and G. Camici, Energy scale(s) and next-to-leading BFKL equation, Phys. Lett. B 430 (1998) 349 [hep-ph/9803389] [INSPIRE].

[39] V.S. Fadin and L. Lipatov, BFKL Pomeron in the next-to-leading approximation, Phys. Lett. B 429 (1998) 127 [hep-ph/9802290] [INSPIRE].

[40] V. Del Duca and C.R. Schmidt, Dijet production at large rapidity intervals, Phys. Rev. D 49 (1994) 4510 [hep-ph/9311290] [INSPIRE].

[41] W.J. Stirling, Production of jet pairs at large relative rapidity in hadron hadron collisions as a probe of the perturbative Pomeron, Nucl. Phys. B 423 (1994) 56 [hep-ph/9401266] [INSPIRE].

[42] V. Del Duca and C.R. Schmidt, BFKL versus $O\left(\alpha_{S}^{3}\right)$ corrections to large rapidity dijet production, Phys. Rev. D 51 (1995) 2150 [hep-ph/9407359] [INSPIRE].

[43] D0 collaboration, B. Abbott et al., Probing BFKL dynamics in the dijet cross section at large rapidity intervals in $p \bar{p}$ collisions at $\sqrt{s}=1800 \mathrm{GeV}$ and $630 \mathrm{GeV}$,

Phys. Rev. Lett. 84 (2000) 5722 [hep-ex/9912032] [INSPIRE].

[44] L.H. Orr and W.J. Stirling, Dijet production at hadron hadron colliders in the BFKL approach, Phys. Rev. D 56 (1997) 5875 [hep-ph/9706529] [INSPIRE].

[45] J. Kwiecinski, A.D. Martin, L. Motyka and J. Outhwaite, Azimuthal decorrelation of forward and backward jets at the Tevatron, Phys. Lett. B 514 (2001) 355 [hep-ph/0105039] [INSPIRE].

[46] W. Giele, E.N. Glover and D.A. Kosower, Higher order corrections to jet cross-sections in hadron colliders, Nucl. Phys. B 403 (1993) 633 [hep-ph/9302225] [INSPIRE].

[47] G. Marchesini et al., HERWIG: a Monte Carlo event generator for simulating hadron emission reactions with interfering gluons. Version 5.1 - April 1991, Comput. Phys. Commun. 67 (1992) 465 [InSPIRE].

[48] A. Sabio Vera, The effect of NLO conformal spins in azimuthal angle decorrelation of jet pairs, Nucl. Phys. B 746 (2006) 1 [hep-ph/0602250] [INSPIRE].

[49] A. Sabio Vera and F. Schwennsen, The azimuthal decorrelation of jets widely separated in rapidity as a test of the BFKL kernel, Nucl. Phys. B 776 (2007) 170 [hep-ph/0702158] [INSPIRE].

[50] C. Marquet and C. Royon, Azimuthal decorrelation of Mueller-Navelet jets at the Tevatron and the LHC, Phys. Rev. D 79 (2009) 034028 [arXiv:0704.3409] [INSPIRE]. 
[51] D. Colferai, F. Schwennsen, L. Szymanowski and S. Wallon, Mueller Navelet jets at LHC complete NLL BFKL calculation, JHEP 12 (2010) 026 [arXiv:1002.1365] [INSPIRE].

[52] J. Bartels, D. Colferai and G. Vacca, The NLO jet vertex for Mueller-Navelet and forward jets: the quark part, Eur. Phys. J. C 24 (2002) 83 [hep-ph/0112283] [INSPIRE].

[53] J. Bartels, D. Colferai and G. Vacca, The NLO jet vertex for Mueller-Navelet and forward jets: the gluon part, Eur. Phys. J. C 29 (2003) 235 [hep-ph/0206290] [INSPIRE].

[54] P. Aurenche, R. Basu and M. Fontannaz, Jet-jet and hadron-jet correlations in hadro- and electro-production, Eur. Phys. J. C 57 (2008) 681 [arXiv:0807.2133] [INSPIRE].

[55] F. Caporale, D.Y. Ivanov, B. Murdaca, A. Papa and A. Perri, The next-to-leading order jet vertex for Mueller-Navelet and forward jets revisited, JHEP 02 (2012) 101 [arXiv: 1112.3752] [INSPIRE].

[56] A. Kotikov and L. Lipatov, NLO corrections to the BFKL equation in QCD and in supersymmetric gauge theories, Nucl. Phys. B 582 (2000) 19 [hep-ph/0004008] [INSPIRE].

[57] F. Schwennsen, Phenomenology of jet physics in the BFKL formalism at NLO, hep-ph/0703198 [INSPIRE].

[58] A. Martin, W. Stirling, R. Thorne and G. Watt, Parton distributions for the LHC, Eur. Phys. J. C 63 (2009) 189 [arXiv:0901.0002] [InSPIRE].

[59] M. Whalley, D. Bourilkov and R. Group, The Les Houches accord PDFs (LHAPDF) and LHAGLUE, hep-ph/0508110 [INSPIRE].

[60] G. Salam, A resummation of large subleading corrections at small $x$, JHEP 07 (1998) 019 [hep-ph/9806482] [INSPIRE].

[61] M. Ciafaloni and D. Colferai, The BFKL equation at next-to-leading level and beyond, Phys. Lett. B 452 (1999) 372 [hep-ph/9812366] [INSPIRE].

[62] M. Ciafaloni, D. Colferai and G. Salam, Renormalization group improved small x equation, Phys. Rev. D 60 (1999) 114036 [hep-ph/9905566] [INSPIRE].

[63] M. Ciafaloni, D. Colferai, G. Salam and A. Stasto, Renormalization group improved small $x$ Green's function, Phys. Rev. D 68 (2003) 114003 [hep-ph/0307188] [INSPIRE].

[64] B. Ducloué, L. Szymanowski and S. Wallon, in preparation.

[65] J. Andersen, V. Del Duca, S. Frixione, C. Schmidt and W.J. Stirling, Mueller-Navelet jets at hadron colliders, JHEP 02 (2001) 007 [hep-ph/0101180] [INSPIRE].

[66] M. Fontannaz, J. Guillet and G. Heinrich, Is a large intrinsic $k_{T}$ needed to describe photon + jet photoproduction at HERA?, Eur. Phys. J. C 22 (2001) 303 [hep-ph/0107262] [InSPIRE].

[67] D.Y. Ivanov and A. Papa, The next-to-leading order forward jet vertex in the small-cone approximation, JHEP 05 (2012) 086 [arXiv: 1202.1082] [INSPIRE].

[68] F. Caporale, D.Y. Ivanov, B. Murdaca and A. Papa, Mueller-Navelet small-cone jets at LHC in next-to-leading BFKL, arXiv:1211.7225 [INSPIRE].

[69] V.T. Kim and G.B. Pivovarov, BFKL QCD Pomeron in high-energy hadron collisions: inclusive dijet production, Phys. Rev. D 53 (1996) 6 [hep-ph/9506381] [INSPIRE].

[70] V.T. Kim and G.B. Pivovarov, New effective Feynman-like rules for the multi-Regge QCD asymptotics of inclusive multi-jet production, Phys. Rev. D 54 (1996) 725 [hep-ph/9601335] [INSPIRE]. 
[71] M. Diehl, D. Ostermeier and A. Schafer, Elements of a theory for multiparton interactions in QCD, JHEP 03 (2012) 089 [arXiv:1111.0910] [INSPIRE].

[72] E. Levin and J. Miller, Two parton shower background for associate W Higgs production, Eur. Phys. J. C 61 (2009) 1 [arXiv:0803.0646] [INSPIRE].

[73] J. Bartels and M. Ryskin, Recombination within multi-chain contributions in pp scattering, arXiv:1105.1638 [INSPIRE].

[74] STAR collaboration, J. Adams et al., Minijet deformation and charge-independent angular correlations on momentum subspace $(\eta, \phi)$ in Au-Au collisions at $\sqrt{s_{N N}}=130 \mathrm{GeV}$, Phys. Rev. C 73 (2006) 064907 [nucl-ex/0411003] [INSPIRE].

[75] PHOBOS collaboration, B. Alver et al., System size dependence of cluster properties from two-particle angular correlations in $\mathrm{Cu}+\mathrm{Cu}$ and $\mathrm{Au}+\mathrm{Au}$ collisions at $\sqrt{s_{N N}}=200 \mathrm{GeV}$, Phys. Rev. C 81 (2010) 024904 [arXiv:0812.1172] [INSPIRE].

[76] STAR collaboration, B. Abelev et al., Long range rapidity correlations and jet production in high energy nuclear collisions, Phys. Rev. C 80 (2009) 064912 [arXiv:0909.0191] [INSPIRE].

[77] PHOBOS collaboration, B. Alver et al., High transverse momentum triggered correlations over a large pseudorapidity acceptance in Au+Au collisions at $\sqrt{s_{N N}}=200 \mathrm{GeV}$, Phys. Rev. Lett. 104 (2010) 062301 [arXiv:0903.2811] [INSPIRE].

[78] CMS collaboration, Observation of long-range near-side angular correlations in proton-proton collisions at the LHC, JHEP 09 (2010) 091 [arXiv: 1009.4122] [INSPIRE].

[79] CMS collaboration, Long-range and short-range dihadron angular correlations in central $\mathrm{Pb}-\mathrm{Pb}$ collisions at a nucleon-nucleon center of mass energy of $2.76 \mathrm{TeV}$,

JHEP 07 (2011) 076 [arXiv: 1105.2438] [INSPIRE].

[80] STAR collaboration, G. Agakishiev et al., Anomalous centrality evolution of two-particle angular correlations from Au-Au collisions at $\sqrt{s_{\mathrm{NN}}}=62$ and $200 \mathrm{GeV}$,

Phys. Rev. C 86 (2012) 064902 [arXiv:1109.4380] [INSPIRE].

[81] ATLAS collaboration, Measurement of the azimuthal anisotropy for charged particle production in $\sqrt{s_{N N}}=2.76 \mathrm{TeV}$ lead-lead collisions with the ATLAS detector, Phys. Rev. C 86 (2012) 014907 [arXiv: 1203.3087] [INSPIRE].

[82] ALICE collaboration, Anisotropic flow of charged hadrons, pions and (anti-)protons measured at high transverse momentum in $\mathrm{Pb}-\mathrm{Pb}$ collisions at $\sqrt{s_{N N}}=2.76 \mathrm{TeV}$, Phys. Lett. B 719 (2013) 18 [arXiv:1205.5761] [inSPIRE].

[83] ATLAS collaboration, Observation of associated near-side and away-side long-range correlations in $\sqrt{s_{N N}}=5.02 \mathrm{TeV}$ proton-lead collisions with the ATLAS detector, arXiv: 1212.5198 [INSPIRE].

[84] ALICE collaboration, Long-range angular correlations on the near and away side in $p$ - $\mathrm{Pb}$ collisions at $\sqrt{s_{N N}}=5.02 \mathrm{TeV}$, Phys. Lett. B 719 (2013) 29 [arXiv:1212.2001] [INSPIRE].

[85] CMS collaboration, Observation of long-range near-side angular correlations in proton-lead collisions at the LHC, Phys. Lett. B 718 (2013) 795 [arXiv:1210.5482] [INSPIRE].

[86] CMS collaboration, Centrality dependence of dihadron correlations and azimuthal anisotropy harmonics in Pb-Pb collisions at $\sqrt{s_{N N}}=2.76 \mathrm{TeV}$, Eur. Phys. J. C 72 (2012) 2012 [arXiv:1201.3158] [INSPIRE]. 
[87] A. Dumitru et al., The ridge in proton-proton collisions at the $\mathrm{LHC}$, Phys. Lett. B 697 (2011) 21 [arXiv: 1009.5295] [INSPIRE].

[88] K. Dusling and R. Venugopalan, Azimuthal collimation of long range rapidity correlations by strong color fields in high multiplicity hadron-hadron collisions, Phys. Rev. Lett. 108 (2012) 262001 [arXiv:1201.2658] [INSPIRE].

[89] A. Kovner and M. Lublinsky, Angular correlations in gluon production at high energy, Phys. Rev. D 83 (2011) 034017 [arXiv: 1012.3398] [InSPIRE].

[90] E. Levin and A.H. Rezaeian, The ridge from the BFKL evolution and beyond, Phys. Rev. D 84 (2011) 034031 [arXiv:1105.3275] [INSPIRE].

[91] K. Dusling and R. Venugopalan, Evidence for BFKL and saturation dynamics from di-hadron spectra at the $L H C$, arXiv:1210.3890 [INSPIRE].

[92] K. Dusling and R. Venugopalan, Explanation of systematics of CMS p+Pb high multiplicity di-hadron data at $\sqrt{s}_{\mathrm{NN}}=5.02 \mathrm{TeV}$, arXiv:1211.3701 [INSPIRE].

[93] K. Dusling and R. Venugopalan, Comparison of the color glass condensate to di-hadron correlations in proton-proton and proton-nucleus collisions, arXiv:1302.7018 [INSPIRE]. 